\title{
High-temperature metamorphism during extreme thinning of the continental crust: a reappraisal of the North Pyrenean passive paleomargin
}

\author{
C. Clerc ${ }^{1,2, *, * *}$, A. Lahfid ${ }^{3}$, P. Monié ${ }^{2}$, Y. Lagabrielle ${ }^{4}$, C. Chopin ${ }^{1}$, M. Poujol ${ }^{4}$, P. Boulvais ${ }^{4}$, J.-C. Ringenbach ${ }^{5}$, \\ E. Masini ${ }^{5}$, and M. de St Blanquat ${ }^{6}$ \\ ${ }^{1}$ Laboratoire de Géologie, CNRS-UMR8538, Ecole normale supérieure, 24 rue Lhomond, 75231 Paris, France \\ ${ }^{2}$ Géosciences Montpellier, CNRS-UMR 5243, Université de Montpellier 2, Place Eugène Bataillon, \\ 34095 Montpellier, France \\ ${ }^{3}$ BRGM/ISTO, 3 avenue Claude Guillemin, 45000 Orléans, France \\ ${ }^{4}$ Géosciences Rennes, UMR 6118, Université Rennes 1, Campus de Beaulieu, \\ 35042 Rennes, France \\ ${ }^{5}$ TOTAL, CSTJF, avenue Larribau, 64000 Pau, France \\ ${ }^{6}$ GET, 14 avenue Edouard Belin, 31400 Toulouse, France \\ *now at: ISTO, 1A rue de la Férollerie, 45100 Orléans, France \\ ** Invited contribution by C. Clerc, recipient of the EGU Outstanding Student Poster (OSP) Award 2012.
}

Correspondence to: C. Clerc (camille.clerc@ cnrs-orleans.fr)

Received: 12 January 2015 - Published in Solid Earth Discuss.: 20 February 2015

Revised: 13 April 2015 - Accepted: 16 May 2015 - Published: 8 June 2015

\begin{abstract}
An increasing number of field examples in mountain belts show that the formation of passive margins during extreme continent thinning may occur under conditions of high to very high thermal gradient beneath a thin cover of syn-rift sediments. Orogenic belts resulting from the tectonic inversion of distal margins and regions of exhumed continental mantle may exhibit high-temperature, low-pressure (HTLP) metamorphism and coeval syn-extensional, ductile deformation. Recent studies have shown that the northern flank of the Pyrenean belt, especially the North Pyrenean Zone, is one of the best examples of such inverted hot, passive margin. In this study, we provide a map of HT-LP metamorphism based on a data set of more than 100 peak-temperature estimates obtained using Raman spectroscopy of the carbonaceous material (RSCM). This data set is completed by previous PT (pressure and temperature) estimates based on mineral assemblages, and new ${ }^{40} \mathrm{Ar}-{ }^{39} \mathrm{Ar}$ (amphibole, micas) and $\mathrm{U}-\mathrm{Pb}$ (titanite) ages from metamorphic and magmatic rocks of the North Pyrenean Zone. The implications on the geological evolution of the Cretaceous Pyrenean paleomargins are discussed. Ages range mainly from 110 to $90 \mathrm{Ma}$,
\end{abstract}

and no westward or eastward propagation of the metamorphism and magmatism can be clearly identified. In contrast, the new data reveal a progressive propagation of the thermal anomaly from the base to the surface of the continental crust. Focusing on the key localities of the Mauléon basin, Arguenos-Moncaup, Lherz, Boucheville and the Bas-Agly, we analyze the thermal conditions prevailing during the Cretaceous crustal thinning. The results are synthetized into a series of three regional thematic maps and into two detailed maps of the Arguenos-Moncaup and Lherz areas. The results indicate a first-order control of the thermal gradient by the intensity of crustal thinning. The highest grades of metamorphism are intimately associated with the areas where subcontinental mantle rocks have been unroofed or exhumed.

\section{Introduction}

Distal domains of present-day passive margins are inaccessible environments from where very little in situ observations have been obtained so far (Sibuet et al., 1979; Ship- 


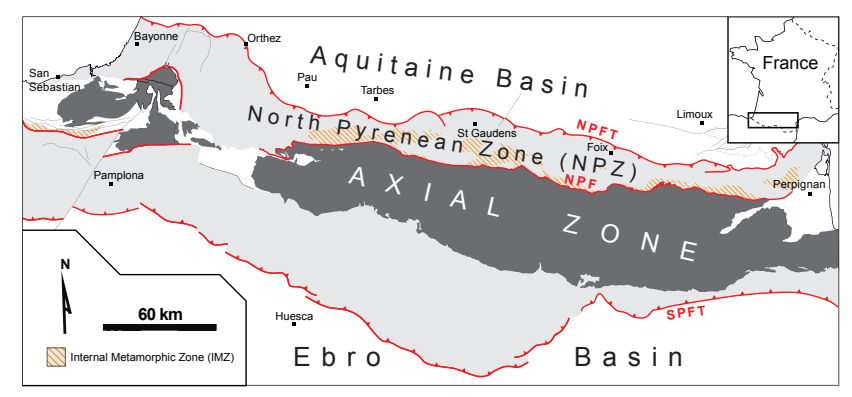

Figure 1. Simplified structural map of the northern flank of the Pyrenean belt. The area between the North Pyrenean Fault (NPF) and the North Pyrenean Frontal Thrust (NPFT) is known as the North Pyrenean Zone (NPZ).

board Scientific Party 1987; Boillot et al., 1987; Sawyer et al., 1994; Whitmarsh et al., 1996, 1998). The understanding of the processes controlling their structural and thermal evolution hence requires the comparison with exhumed analogs of fossil passive margins. The first and moststudied analogs have been the paleomargins exposed along the Alpine arc (Lemoine et al., 1987; Froitzheim and Eberli 1990; Manatschal and Nievergelt 1997; Manatschal 2004). Other fossil margins have recently been identified within the Zagros (Wrobel-Daveau et al., 2010) and AppalachianCaledonian orogenic belts (Andersen et al., 2012; Chew et Van Staal, 2014). At present, the Pyrenean domain is also considered as hosting relevant analogs of distal passive margins, and its pre-orogenic evolution is being intensely revisited (Lagabrielle and Bodinier, 2008; Jammes et al., 2009; Lagabrielle et al., 2010; Masini 2011; Clerc et al., 2012; Clerc et al., 2013; Masini et al., 2014; Tugend et al., 2014).

Unlike the Alpine analog, the North Pyrenean domain did not undergo subduction, and the thermal pattern recorded in the pre- and syn-rift material has not been overprinted by major crustal overthrusts or subductions. The pre-Pyrenean inverted margins hence offer suitable direct access to the thermal imprint of crustal thinning and subsequent continental breakup. Regional high-temperature, low-pressure (HTLP) metamorphism is known along the northern rim of the Pyrenean belt where it developed coevally with a major Cretaceous crustal thinning event leading (Ravier, 1959; Bernus-Maury, 1984; Azambre and Rossy, 1976; Golberg and Leyreloup, 1990; Dauteuil and Ricou, 1989; Clerc and Lagabrielle, 2014). The HT metamorphic rocks, deriving mainly from pre-rift to syn-rift sediments, are distributed in a narrow WNW-ESE belt defined as the North Pyrenean Zone (NPZ), bounded by two major post-metamorphic thrusts, the North Pyrenean Fault (NPF) to the south and the North Pyrenean Frontal Thrust (NPFT) to the north (Fig. 1). The NPZ hosts about 40 outcrops of sub-continental peridotite - among which the Lherz body, lithotype of the lherzolite - whose exhumation is directly attributed to the extreme thinning of the lithosphere (Kornprobst and Vielzeuf, 1984; Lagabrielle and Bodinier, 2008).

At a regional scale, the NPZ is thought to represent the portion of the lithosphere that accommodated most of the deformation during the counterclockwise rotation of the Iberian plate with respect to the Europa plate in the mid-Cretaceous (Choukroune and Mattauer, 1978). The zone affected by the HT-LP metamorphism is characterized by an intense ductile deformation - S1 of Choukroune (1972); Choukroune (1976) - first attributed to compression and later reinterpreted as resulting from syn-extensional or transtensional deformation (Golberg 1987; Golberg and Leyreloup, 1990; Lagabrielle et al., 2010; Clerc and Lagabrielle, 2014).

Several estimates of peak temperatures have been published (Golberg and Leyreloup 1990, 1990; Vauchez et al., 2013), but no comprehensive metamorphic map is available so far at the scale of the whole NPZ.

In this study, we provide a map of the HT Pyrenean metamorphism based on a data set of more than 100 peaktemperature estimates obtained using Raman spectroscopy of the carbonaceous material (RSCM) on samples collected during the past 5 years along the whole NPZ (Fig. 2). From east to west the sampled regions are the Bas-Agly basin, the Tarascon and Aulus basins (eastern NPZ), the Ballongue basin (central NPZ) and the Mauléon basin (western NPZ). This data set is completed by previous PT estimates based on analysis of mineral assemblages from the Bas-Agly, Boucheville and Pays de Sault areas by Golberg and Leyreloup (1990). We provide 18 new ${ }^{40} \mathrm{Ar}-{ }^{39} \mathrm{Ar}$ (amphibole, micas) ages and $1 \mathrm{U}-\mathrm{Pb}$ (titanite) age from metamorphic and magmatic rocks of the North Pyrenean Zone. We further report some characteristics of the deformation associated with the HT metamorphic imprint, and we discuss the significance of the thermal event in the frame of the extension of the continental crust and local mantle exhumation. Triassic and Jurassic aborted rifting events predated the development of a major Cretaceous crustal thinning event, which culminated in the crustal separation between the Iberia and European plates (Puigdefabregas and Souquet, 1986; Vergés and Garcia-Senz, 2001). Continental rifting in the Pyrenean domain occurred in response to the counterclockwise rotation of Iberia relative to Europe, coeval with the onset of oceanic spreading in the Bay of Biscay between Chron M0 and A33o (approximately 125-83 Ma) (Le Pichon et al., 1970; Choukroune and Mattauer, 1978; Olivet, 1996; Gong et al., 2008; Jammes et al., 2009). After an early rifting episode during the late Aptian, narrow, non-connected Albian basins opened north of the basement of the Pyrenean Axial Zone, along a wide domain opened between Iberia and Europe (Choukroune and Mattauer, 1978; Olivet, 1996; Jammes et al., 2009). They connected together during the Cenomanian when the rift zone became wider and deeper. The main infills of the basins are dark-colored pelites, sandstone, and breccias deposits, referred to as "Flysch noir" or "Flysch ardoisier" in the literature (Debroas, 

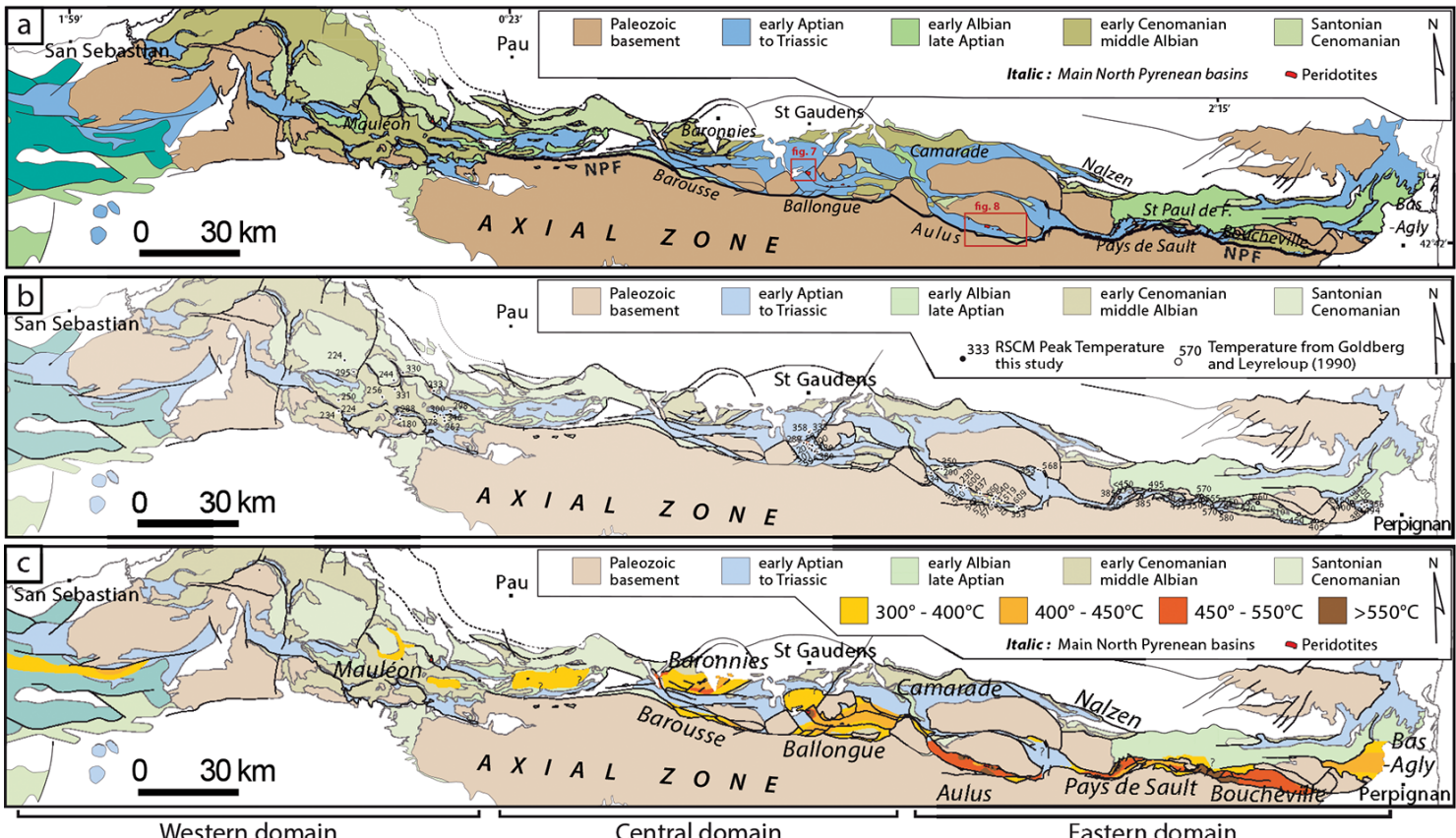

Figure 2. (a) Simplified geological map of the North Pyrenean Zone, compiled from Choukroune and Séguret (1973), Golberg and Leyreloup (1990), Debroas (2003) and Jammes et al. (2009), and from the 1/50000 geological map of the BRGM. (b) Map of peak metamorphic temperatures estimated using RSCM geothermometry (black dots). Only some values are reported on the map. See Table 1 for an exhaustive list and location of all the RSCM data. Temperatures of Golberg and Leyreloup (1990) are also reported for the eastern NPZ (white dots). (c) Map of isometamorphic zones; data compiled are from this study and the literature (Choukroune and Séguret, 1973; Bernus-Maury, 1984; Golberg, 1987; Golberg and Leyreloup, 1990).

1976, 1978, 1990; Souquet et al., 1985). The black flysch is organized into three megasequences (I, II, and III), which are the records of three successive steps in the opening of the basins. Megasequence I corresponds to the opening of narrow half grabens, megasequence II registers the opening of en echelon $10 \mathrm{~km}$ wide basins, and megasequence III records the coalescence of the basins into a large trough with internal and external parts separated by central highs (Debroas, 1990).

The kinematics of the Iberian plate during Aptian-Albian and younger Cretaceous times is still strongly debated (Olivet 1996; Sibuet, Srivastava, et Spakman 2004; Jammes et al., 2009; Vissers et Meijer 2012a, b). Three main types are generally opposed: (i) the transtensional rift model (Choukroune et Mattauer 1978; Olivet 1996), which involves a dominant left-lateral strike-slip along the NPF; (ii) a model implying most of the left-lateral movement during the Jurassic to Aptian times followed by orthogonal extension during Albian to Cenomanian times (Schettino et Turco 2010; Jammes et al., 2009); (iii) and a scissor-opening model, which implies the existence of an important subduction beneath the Pyrenean belt (Srivastava et al., 1990; Sibuet et al., 2004; Vissers and Meijer 2012a, b). For these authors, the extension observed in the pre-Pyrenean domain at that time (Dinarès-Turell and Garcia-Senz, 2000, 2002) would result from back-arc extension or gravitational instability of the lithosphere above the subducted oceanic lithosphere. This scenario implies the northward subduction of a large portion of the Neotethyan oceanic domain, which is hardly compatible with current reconstructions of the Alpine Tethys (Manatschal and Bernouilli, 1999; Handy et al., 2010; Schettino and Turco, 2010). Furthermore, recent seismic tomography (Souriau et al., 2008; Chevrot et al., 2014a, b) precluded the existence of a subducted hundredth-of-a-kilometer-long oceanic lithosphere. Instead, they report evidence for the subduction of a thinned Iberian crust to ca. $\sim 70 \mathrm{~km}$ depth.

\section{The HT-LP metamorphism: ages and coeval magmatism}

\subsection{The age of HT-LP metamorphism: a review}

The metasediments of the NPZ are affected by a HT-LP metamorphism with temperatures commonly higher than $600^{\circ} \mathrm{C}$ and pressures lower than $4 \mathrm{kbar}$ (Fig. 2; BernusMaury, 1984; Golberg and Leyreloup, 1990; Vauchez et al., 2013). The NPZ is characterized by strong temperature gradients affecting mostly the pre- and syn-rift Mesozoic sediments, ranging in age from the Trias to the base of the Upper Cretaceous (although the extension of the thermal anomaly to Paleozoic material has rarely been tested). The youngest 


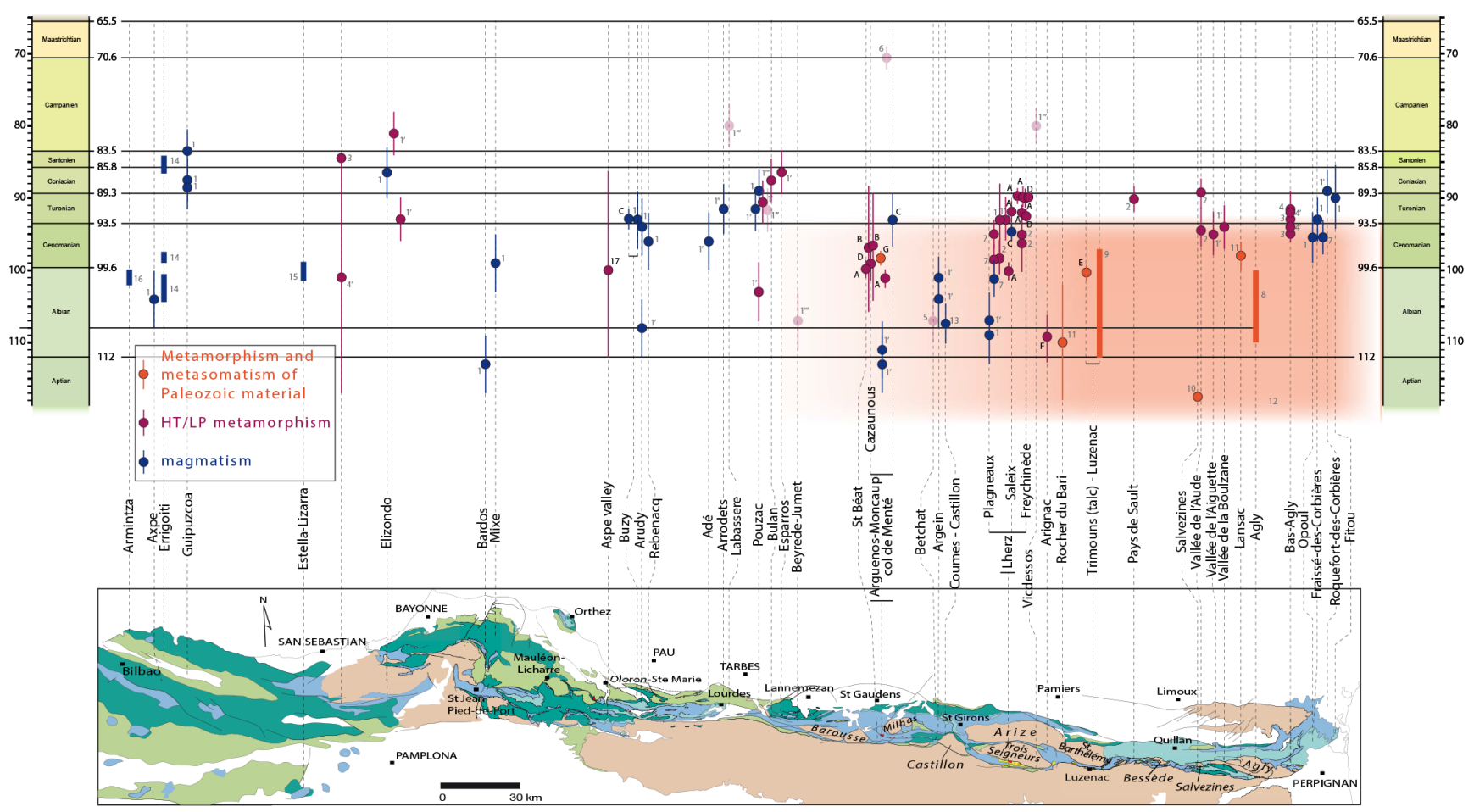

Figure 3. Compilation of the age and localization of the Cretaceous metamorphism and magmatism, modified and updated from Debroas and Azambre (2012). (1): Montigny et al., 1986; ${ }^{40} \mathrm{~K}-{ }^{40} \mathrm{Ar}$ on amphiboles from magmatic and metamorphic rocks. (1'): Montigny et al., 1986; ${ }^{40} \mathrm{~K}-{ }^{40} \mathrm{Ar}$ on micas. (1"): Montigny et al., $1986 ;{ }^{40} \mathrm{~K}-{ }^{40} \mathrm{Ar}$ on feldspath(oids). (1")): Montigny et al., $1986 ;{ }^{40} \mathrm{~K}-{ }^{40} \mathrm{Ar}$ on bulk rocks. (2): Golberg and Maluski, 1988. (3): Albarède and Michard-Vitrac, 1978; ${ }^{40} \mathrm{Ar}-{ }^{39} \mathrm{Ar}$ on phlogopite from metasediments. (4): Albarède and Michard-Vitrac, 1978; ${ }^{40} \mathrm{Ar}-{ }^{39} \mathrm{Ar}$ on orthoclase from metasediments. (4'): Albarède and Michard-Vitrac, $1978 ;{ }^{87} \mathrm{Rb}-{ }^{87} \mathrm{Sr}$ on phlogopite from metasediments. (5): Thiébaut et al., 1988; ${ }^{40} \mathrm{~K}-{ }^{40} \mathrm{Ar}$ (on bulk rock?) from Triassic meta-evaporites. (6): Hervouët et al., 1987; ${ }^{40} \mathrm{~K}-$ ${ }^{40} \mathrm{Ar}$ on bulk rock (ophite) from the vallée du Job. (7): Golberg et al., 1986; ${ }^{40} \mathrm{Ar}-{ }^{39} \mathrm{Ar}$. (8): Nicolas, 1998, unpublished master thesis; ${ }^{40} \mathrm{Ar}-{ }^{39} \mathrm{Ar}$ on muscovite and biotite from the charnockite and various schists of the Agly Paleozoic massif. (9): Schärer et al., 1999; U-Pb on xenotime and monazite from talc deposit. (10): Boulvais et al., 2007; ${ }^{40} \mathrm{Ar}-{ }^{39} \mathrm{Ar}$ on muscovite from albitite. (11): Poujol et al., 2010; U-Th-Pb on titanite and monazite from albitite. (12): Fallourd et al., 2014; U-Pb on titanite in albitite (faded orange field). (13) Henry et al., 1998; ${ }^{40} \mathrm{Ar}-{ }^{39} \mathrm{Ar}$ on amphibole from amphiclasite. (14): Castanares et al., 1997; 2001; chronostratigraphy of submarine volcanic episodes. (15): Lopez-Horgue et al., 1999; chronostratigraphy of submarine volcanic episodes. (16): Lopez-Horgue et al., 2009; chronostratigraphy of submarine volcanic episodes. (17) Monié, unpublished; ${ }^{40} \mathrm{Ar}-{ }^{39} \mathrm{Ar}$ on Ms from marble. (A): This study; ${ }^{40} \mathrm{Ar}-{ }^{39} \mathrm{Ar}$ on muscovite from marble. (B): This study; ${ }^{40} \mathrm{Ar}-{ }^{39} \mathrm{Ar}$ on amphibole from marble. (C): This study; ${ }^{40} \mathrm{Ar}-{ }^{39} \mathrm{Ar}$ on amphibole from gabbro and teschenite. (D): This study; ${ }^{40} \mathrm{Ar}-{ }^{39} \mathrm{Ar}$ on amphibole from meta-ophite. (E): This study; ${ }^{40} \mathrm{Ar}-{ }^{39} \mathrm{Ar}$ on phlogopite from talc deposit. (F): This study; ${ }^{40} \mathrm{Ar}-$ ${ }^{39} \mathrm{Ar}$ on amphibole in gypsum. (G): This study; $\mathrm{U}-\mathrm{Pb}$ on titanite in albitite dyke. Spots in transparency must be considered with caution since they were obtained with ${ }^{40} \mathrm{~K}-{ }^{40} \mathrm{Ar}$ on bulk rock.

metamorphosed terrains in the NPZ are Santonian in age and were only affected by an epizonal metamorphism (Mattauer, 1964; Choukroune, 1972; Debroas, 1987).

The HT-LP metamorphism has alternatively been related to late-Cretaceous compressive events (Mattauer, 1968; Choukroune, 1976) or to a "pre-Cenomanian crustal fracturation" (Ravier, 1959; Souquet et al., 1977). Choukroune and Mattauer (1978) re-interpreted this thermal event as the consequence of an important thinning of the continental crust for which, due to slow migration of the isotherms, the maximum of the thermal anomaly would only be reached during the initiation of the convergent tectonics.
Since the 1980s, the thermal anomaly responsible for the development of the metamorphic event is attributed to very high geothermal gradients related to an intense crustal and lithospheric thinning episode during the Cretaceous (Vielzeuf and Kornprobst, 1984; Golberg, 1987; Golberg and Maluski, 1988; Dauteuil and Ricou, 1989; Golberg and Leyreloup, 1990). This assumption is based on four main geological features: (i) HT-LP assemblages are recorded along the extensive/transtensive domain represented by the NPZ; (ii) HT-LP metamorphism is contemporaneous with an episode of alkaline magmatism; (iii) HT-LP metasediments are spatially related to tectonically exhumed deep-seated material (granulites and peridotites); and (iv) the metamorphic 


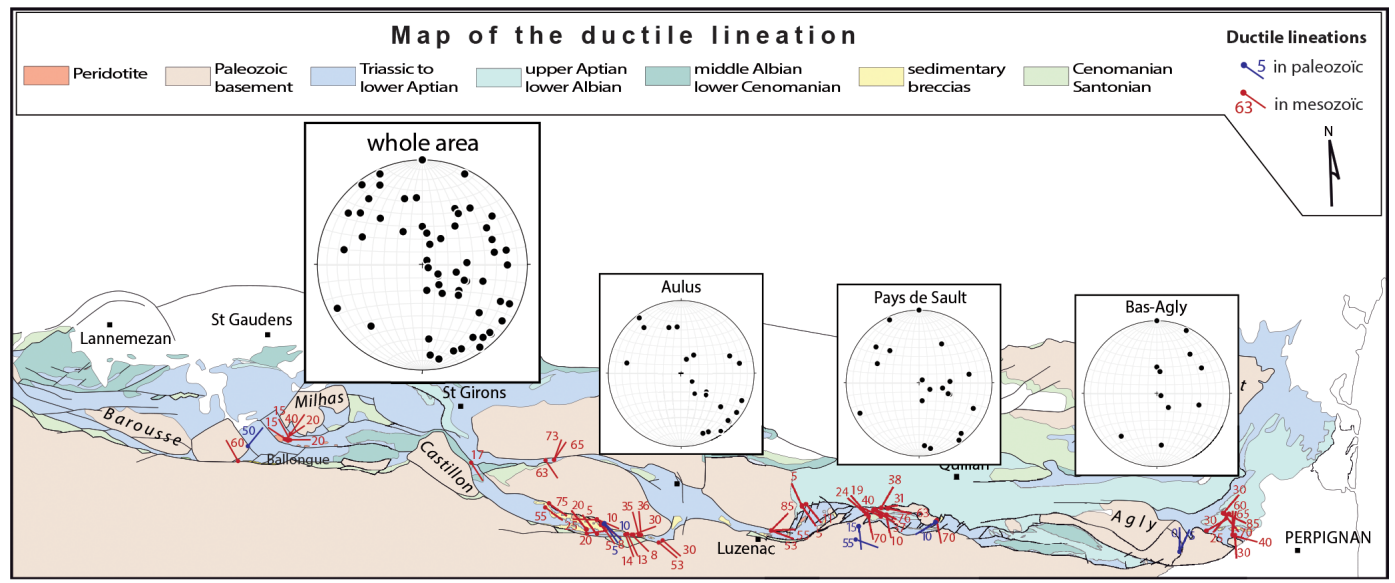

Figure 4. General map of the ductile lineation measured in the NPZ, in the Mesozoic material (blue) and in the Paleozoic basement (red).

event affected Albian-Cenomanian flyschs during and just after their deposition.

Previous geochronological studies (Fig. 3) of the Cretaceous metamorphism of the Pyrenees revealed ages ranging from the Albian to the Santonian, e.g., mainly in the range 105-85 Ma (Albarède and Michard-Vitrac, 1978a; Albarède and Michard-Vitrac, 1978b; Montigny et al., 1986; Golberg and Maluski, 1988; Golberg et al., 1986; Bandet Y. and Gourinard Y. in Thiébaut et al., 1988).

In addition to the HT-LP metamorphism well developed in Mesozoic material, one must also consider the hydrothermal alteration responsible for albitization and dequartzification of the North Pyrenean massifs and the formation of massive talc deposits during the late Aptian to early Cenomanian (Moine et al., 1989; Demange et al., 1999; Schärer et al., 1999; Boulvais et al., 2007; Poujol et al., 2010) at temperatures ranging from $250^{\circ} \mathrm{C}$ (Moine et al., 1989; Boulvais et al., 2006) to more than $500^{\circ} \mathrm{C}$ (sodic-calcic metasomatism; Fallourd et al., 2014). Radiogenic chronometers in the Paleozoic basement of the North Pyrenean massifs have been reset during Cretaceous times (e.g., Costa and Maluski 1988; St Blanquat et al., 1990; Boutin et al., 2015, in the St Barthelemy massif), which is indicative of general heating of the NPZ. Cretaceous ages have also been reported in mylonitized granitoids from the Axial Zone in the eastern Pyrenees (Monié et al., 1994), suggesting that reheating and related deformation propagated further south of the NPF.

According to previous studies of the eastern NPZ, the intensity of the HT-LP metamorphism is thought to be directly related to the magnitude of crustal attenuation, with high-grade metasediments systematically associated with peridotites or granulites, and lower-grade metasediments associated with mid- to upper-crustal units (Golberg and Leyreloup, 1990). Our new data set, extended to the whole NPZ, confirms this trend. To push forward the characterization and significance of the HT-LP Cretaceous metamorphism, we will discuss our results at the scale of the whole
Pyrenean realm, then zooming at the scale of the basin and at the scale of the sedimentary pile.

\section{Metamorphism and deformation: the S1 deformation (Choukroune, 1976) is syn-extensional}

All along the inner NPZ, Triassic to Albian-Cenomanian rocks bear evidence of intense ductile deformation generally transposed on the original stratification of the Mesozoic metasediments. Stretching lineations are marked by the elongated aspect of some marbles and by preferred orientations of the HT-LP minerals (scapolite). A map of the HT ductile lineation of the eastern NPZ is presented in Fig. 4. Despite a relative dispersion probably due to the later compressional movements, the lineation is generally trending along a NWSE direction. Although it may be difficult, the distinction of Mesozoic marbles from Paleozoic (mainly Devonian) ones can be done by comparison with less metamorphosed facies (e.g., Debroas in Ternet et al., 1997); some fossils allow for unambiguous attribution to the Mesozoic series (Fig. 5). Boudinage and folds are frequent and are observed at different scales. Sheath folds and isoclinal folds are commonly observed in both pre-Albian marbles and Albian-Cenomanian metapelites. In the high-grade marbles of the Internal Metamorphic Zone (IMZ; e.g., Aulus and Arguenos-Moncaup areas), isoclinal folds are commonly refolded and show complex 3-D geometries. Such characteristics are evocative of flow folds (viscous folds without visible axial-plane cleavage or foliation; Wynne-Edwards, 1963).

The analysis of metamorphic parageneses indicates that the metamorphism can be both static (Ravier, 1959) and synkinematic (Bernus-Maury, 1984). According to Choukroune (1976), the long-lasting Cretaceous metamorphism is contemporaneous with a first phase of deformation (S1) and continues during a part of a second phase (S2 of Choukroune, 1976). 

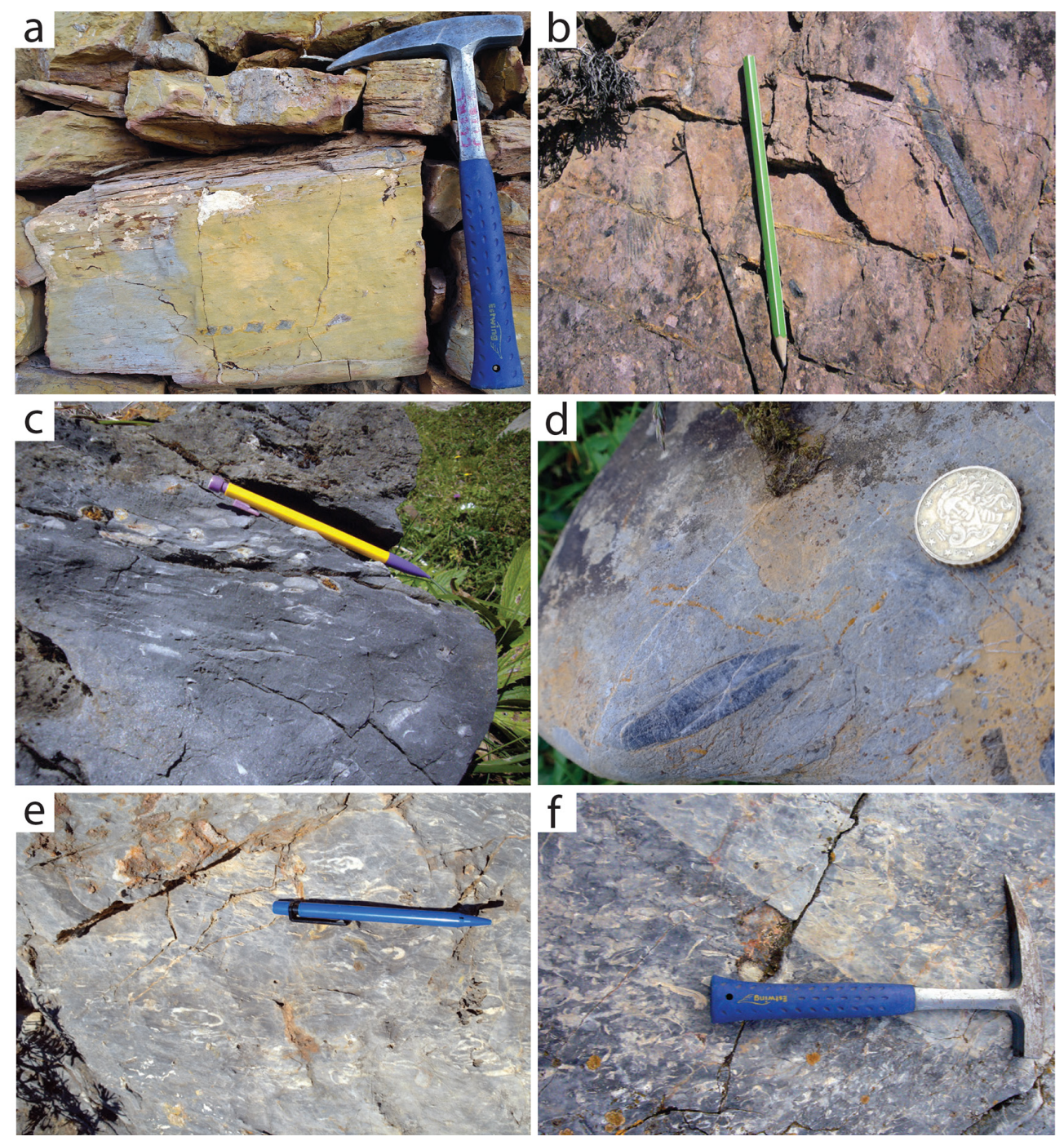

Figure 5. Examples of deformed Mesozoic fossils from the NPZ. Boudinaged (a) and elongated (b) belemnites and pecten in the Bas-Agly. Ductiley deformed bivalves and belemnites at Port de Saleix (c) and Col Dret (d). Deformed Urgonian rudists in the Bas-Agly (e and f).

Boudinage of dolomitic and silicic layers is commonly observed within the marbles due to their rheological contrast with the calcitic host. The boudins and the boudin necks form symmetric lenses displaying a "chocolate tablet" aspect (Vauchez et al., 2013). The synchronicity between deformation and metamorphism has also been demonstrated by the HT fabrics of calcite in the marble of the IMZ (Vauchez et al., 2013; Lagabrielle et al., 2015).

Considering that the onset of the convergence in the Pyrenean realm is estimated to occur during the Santonian (Garrido-Megias and Rios, 1972; McClay et al., 2004), the pre-Santonian radiometric ages obtained for the HT metamorphism are in agreement with a pre-convergence event (Fig. 3).

\section{Magmatism}

The North Pyrenean realm is affected by moderate but welldistributed magmatic activity during the Mesozoic, responsible for the emplacement of two main groups of rocks: (i) tholeitic dolerites of Triassic age, generally referred to as "ophites" (Montigny et al., 1982; Azambre et al., 1987), and (ii) a wide variety of small intrusive and effusive Cretaceous alkaline magmatic rocks (Montigny et al., 1986; Azambre et al., 1992; Rossy et al., 1992). The age of the Cretaceous magmatism is often constrained by stratigraphic correlations with the sedimentary formations in which volcanic or intrusive rocks are observed (Fig. 3; San Miguel de la Camara 1952; Rossy 1988; Castanares et al., 1997; López-Horgue et al., 1999; Castanares et al., 2001; López-Horgue et al., 2009). In Buzy (Pyrénées-Atlantiques), teschenites outcrop as sills intrusive in the Turonian flysch where they developed 
a hornblende-hornfels contact metamorphism aureole (Casteras et al., 1970). In the Cantabrian basin, thick formations of lava flows with pillows are interbedded within the upper Albian to Santonian sedimentary formations (Castanares et al., 1997, 2001; Carracedo et al., 1999). In addition, absolute ages are available on Cretaceous magmatic rocks, with a spread from 113 to $85 \mathrm{Ma}$ (Fig. 3; Golberg et al., 1986; Montigny et al., 1986; Henry et al.,1998). Some authors distinguish an early phase of intrusive magmatism followed by a paroxysm from the Cenomanian to the end of the Turonian (Debroas and Azambre, 2012). Some manifestations of the Cretaceous alkaline magmatism have also been recognized within the peridotite bodies of the NPZ, where they appear as dykes of amphibole-bearing pyroxenites and hornblendes ("ariégites à amphiboles" and "lherzites" of Lacroix 1917) considered to represent trans-mantellic melt conduits for the Cretaceous alkaline magmatism (Bodinier et al., 1987; Conquéré 1971; Vétil et al., 1988). Radiometric dating of these rocks gave ages around 100 Ma (Vershure et al., 1967; Golberg et al., 1986; Henry et al., 1998). The peridotite bodies of Tuc de Desse, Arguenos-Moncaup, Montaut and Turon de la Técouère also contain gabbroic intrusions that present chilled margins along their contacts, indicating that some of the mantle rocks were already cooled during the emplacement of the magmatic rocks (Azambre and Monchoux, 1998).

\section{Raman spectroscopy of carbonaceous materials: analytical method and thermometry}

Raman spectroscopy has been used successfully to characterize the structural evolution of carbonaceous material (CM), reflecting a transformation from disordered to well-ordered CM during metamorphism (Wopenka and Pasteris, 1993). The irreversible polymerization and reorganization of these materials is reflected in their Raman spectrum by the decreasing width of the graphite $\mathrm{G}$ band and gradual disappearance of the defect bands, first D3 and D4, then D1 and D2. The Raman spectrum of well-ordered CM (perfect graphite) contains only the $\mathrm{G}$ band. This spectral evolution with increasing graphitization was related to temperature and quantified, providing a means to determine peak temperatures attained by metamorphic rocks (Beyssac et al., 2002). This is the basis of the RSCM geothermometer, which was calibrated in the range $330-650^{\circ} \mathrm{C}$ by Beyssac et al. (2002) and extended to the range $200-320^{\circ} \mathrm{C}$ by Lahfid et al. (2010). In this study, we have applied these two calibrations to estimate paleotemperatures in marbles and pelitic-psammitic metasedimentary rocks from the Paleozoic to Upper Cretaceous series of the NPZ.

Raman analyses were performed using a Renishaw (Wotton-under-Edge, UK) InVIA Reflex microspectrometer at ENS Paris. Before each session, the spectrometer was calibrated with silicon standard. The light source was a $514 \mathrm{~nm}$ Spectra Physics argon laser. The output laser power is around
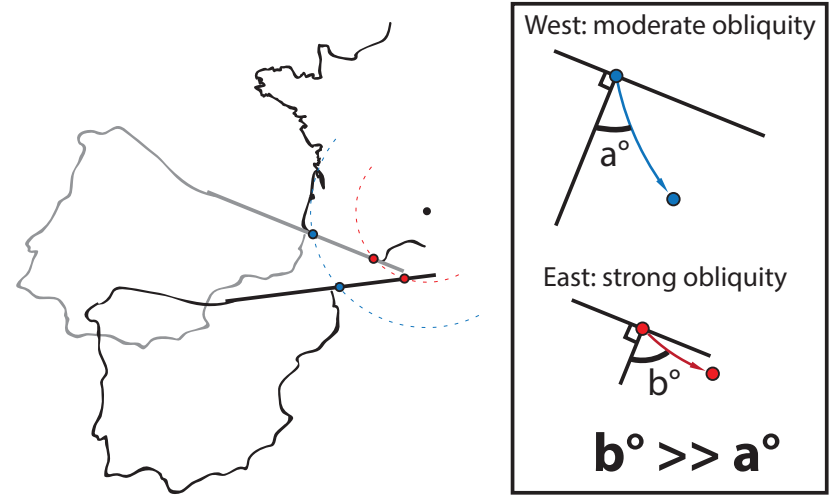

Figure 6. Example of variable mode of extension along a single linear plate boundary. In this example, the eastern domain of the future NPZ undergoes transtension with a strong left-lateral obliquity, while the western domain opens with a moderate obliquity.

$20 \mathrm{~mW}$, but only around $1 \mathrm{~mW}$ reached the surface sample through the DMLM Leica (Wetzlar, Germany) microscope with a $100 \times$ objective $(\mathrm{NA}=0.90)$. Edge filters eliminated the Rayleigh diffusion, and the Raman light was dispersed using a $1800 \mathrm{~g} \mathrm{~mm}^{-1}$ grating before being analyzed by a Peltier-cooled RENCAM CCD detector. Measurements were done on polished thin sections cut normal to the foliation and parallel to the lineation ( $x z$ structural plane). To avoid the effect of polishing on the CM structural state, the CM particles analyzed were below a transparent adjacent mineral, usually calcite or quartz. The results are presented in Table 1 and Figs. 2, 7 and 8.

\section{Geochronology}

In order to corroborate previous results and to extend the data set of the ages of the North Pyrenean magmatism and metamorphism, we selected 19 samples from the NPZ. We focused on the marbles and gabbros of the key localities of Lherz (LHZ samples) and Arguenos-Moncaup (MP samples). Eighteen metamorphic and magmatic samples were dated using ${ }^{40} \mathrm{Ar}-{ }^{39} \mathrm{Ar}$ step heating of muscovite, phlogopite and amphibole, and a sample of albitite vein cross-cutting Paleozoic material has been dated by $\mathrm{U}-\mathrm{Pb}$ on titanite.

\section{$6.1{ }^{40} \mathrm{Ar}-{ }^{39} \mathrm{Ar}$ dating of metamorphic and magmatic samples from the NPZ}

Fresh samples were selected in the field for step-heating laser probe ${ }^{40} \mathrm{Ar}-{ }^{39} \mathrm{Ar}$ dating. The samples were crushed and sieved, and single grains of micas (biotite and muscovite) and amphibole were handpicked under binocular microscope and cleaned in an ultrasonic bath using acetone and distilled water. Micas and amphiboles were packaged in aluminium foils and irradiated in the core of the TRIGA Mark II nuclear reactor of Pavia (Italia) with several aliquots of the Fish Canyon 
Table 1. RSCM peak temperatures from the NPZ. The parameters RA1 $1_{\text {Lahfid }}$ (Lahfid et al., 2010) and R2 $2_{\text {Beyssac }}$ (Beyssac et al., 2002) are used to estimate temperatures $<320$ and $>330^{\circ} \mathrm{C}$, respectively. RA1 $1_{\text {Lahfid }}, \mathrm{R} 2_{\text {Beyssac }}$ and $T$ are expressed in terms of mean value and SD of all the data obtained within each of the 106 samples from the NPZ sample. Standard errors $\varepsilon$ are given for temperatures $(=1 \sigma$ SD divided by the square root of the number of measurements).

\begin{tabular}{|c|c|c|c|c|c|c|c|c|c|c|}
\hline \multirow{2}{*}{$\begin{array}{l}\text { Sample } \\
\text { FO2a }\end{array}$} & \multirow{2}{*}{$\begin{array}{l}\text { Location } \\
\text { Oust }\end{array}$} & \multirow{2}{*}{$\begin{array}{l}\text { Lithology } \\
\text { Upper Albian }\end{array}$} & \multicolumn{2}{|c|}{ Position } & \multirow{2}{*}{$\begin{array}{l}\mathrm{Nb} \\
\text { Sprectra }\end{array}$} & \multirow{2}{*}{$\begin{array}{l}\text { Method } \\
\text { B }\end{array}$} & \multirow{2}{*}{$\begin{array}{l}\begin{array}{l}\text { Raman } \\
\text { parameter }\end{array} \\
0.66\end{array}$} & \multirow{2}{*}{$\begin{array}{l}\text { SD } \\
0.01\end{array}$} & \multirow{2}{*}{$\begin{array}{l}\begin{array}{l}\mathrm{T} \\
\left({ }^{\circ} \mathrm{C}\right)\end{array} \\
349\end{array}$} & \multirow{2}{*}{$\begin{array}{l}\mathrm{SE} \\
\left({ }^{\circ} \mathrm{C}\right)\end{array}$} \\
\hline & & & $42^{\circ} 53^{\prime} 41.17^{\prime \prime} \mathrm{N}$ & $1^{\circ} 12^{\prime} 55.27^{\prime \prime} \mathrm{E}$ & & & & & & \\
\hline $\mathrm{FO} 2 \mathrm{~b}$ & Oust & Upper Albian & $42^{\circ} 53^{\prime} 41.17^{\prime \prime} \mathrm{N}$ & $1^{\circ} 12^{\prime} 55.27^{\prime \prime} \mathrm{E}$ & 18 & $\mathrm{~B}$ & 0.65 & 0.01 & 353 & 1 \\
\hline ERC1 & Ercée & Turonian-Senonian & $42^{\circ} 49^{\prime} 56.67^{\prime \prime} \mathrm{N}$ & $1^{\circ} 17^{\prime} 49.02^{\prime \prime} \mathrm{E}$ & 13 & $\mathrm{~L}$ & 0.56 & 0.008 & 242 & 3 \\
\hline ERC3 & Ercée & Turonian-Senonian & $42^{\circ} 49^{\prime} 55.00^{\prime \prime} \mathrm{N}$ & $1^{\circ} 17^{\prime} 51.42^{\prime \prime} \mathrm{E}$ & 10 & $\mathrm{~L}$ & 0.56 & 0.005 & 238 & 2 \\
\hline ERC4 & Ercée & Turonian-Senonian & $42^{\circ} 49^{\prime} 55.00^{\prime \prime} \mathrm{N}$ & $1^{\circ} 17^{\prime} 51.42^{\prime \prime} \mathrm{E}$ & 11 & $\mathrm{~L}$ & 0.56 & 0.011 & 242 & 4 \\
\hline ERC5 & Ercée & Turonian-Senonian & $42^{\circ} 49^{\prime} 54.69^{\prime \prime} \mathrm{N}$ & $1^{\circ} 17^{\prime} 51.73^{\prime \prime} \mathrm{E}$ & 11 & $\mathrm{~L}$ & 0.55 & 0.01 & 222 & 4 \\
\hline ERC6c & Ercée & Turonian-Senonian & $42^{\circ} 49^{\prime} 53.81^{\prime \prime} \mathrm{N}$ & $1^{\circ} 17^{\prime} 52.71^{\prime \prime} \mathrm{E}$ & 11 & $\mathrm{~L}$ & 0.55 & 0.01 & 219 & 5 \\
\hline ERC8 & Ercée & Barremian & $42^{\circ} 49^{\prime} 49.23^{\prime \prime} \mathrm{N}$ & $1^{\circ} 17^{\prime} 51.84^{\prime \prime} \mathrm{E}$ & 13 & B & 0.21 & 0.07 & 543 & 6 \\
\hline ERC9 & Ercée & Barremian & $42^{\circ} 49^{\prime} 46.36^{\prime \prime} \mathrm{N}$ & $1^{\circ} 17^{\prime} 48.48^{\prime \prime} \mathrm{E}$ & 10 & B & 0.18 & 0.05 & 560 & 8 \\
\hline ERC13 & Ercée & Barremian & $42^{\circ} 49^{\prime} 35.00^{\prime \prime} \mathrm{N}$ & $1^{\circ} 17^{\prime} 32.10^{\prime \prime} \mathrm{E}$ & 11 & $\mathrm{~B}$ & 0.09 & 0.07 & 597 & 9 \\
\hline ERC14 & Ercée & Barremian & $42^{\circ} 49^{\prime} 23.18^{\prime \prime} \mathrm{N}$ & $1^{\circ} 17^{\prime} 48.49^{\prime \prime} \mathrm{E}$ & 18 & $\mathrm{~B}$ & 0.04 & 0.06 & 620 & 6 \\
\hline ERC14a & Ercée & Barremian & $42^{\circ} 49^{\prime} 23.18^{\prime \prime} \mathrm{N}$ & $1^{\circ} 17^{\prime} 48.49^{\prime \prime} \mathrm{E}$ & 10 & $\mathrm{~B}$ & 0.19 & 0.06 & 555 & 9 \\
\hline ERC16 & Ercée & Aptian & $42^{\circ} 49^{\prime} 31.90^{\prime \prime} \mathrm{N}$ & $1^{\circ} 17^{\prime} 35.23^{\prime \prime} \mathrm{E}$ & 10 & B & 0.2 & 0.08 & 551 & 11 \\
\hline ERC17 & Ercée & Berriasian-Hauterivian & $42^{\circ} 49^{\prime} 37.08^{\prime \prime} \mathrm{N}$ & $1^{\circ} 18^{\prime} 46.17^{\prime \prime} \mathrm{E}$ & 11 & $\mathrm{~B}$ & 0.15 & 0.1 & 578 & 9 \\
\hline ERC19 & Ercée & Barremian & $42^{\circ} 49^{\prime} 29.06^{\prime \prime} \mathrm{N}$ & $1^{\circ} 18^{\prime} 44.60^{\prime \prime} \mathrm{E}$ & 9 & B & 0.25 & 0.07 & 531 & 10 \\
\hline ERC20 & Sentenac d'Oust & Albian & $42^{\circ} 52^{\prime} 36.45^{\prime \prime} \mathrm{N}$ & $1^{\circ} 10^{\prime} 9.52^{\prime \prime} \mathrm{E}$ & 14 & $\mathrm{~B}$ & 0.24 & 0.04 & 534 & 6 \\
\hline ERC21a & Sentenac d'Oust & Albian & $42^{\circ} 52^{\prime} 35.49^{\prime \prime} \mathrm{N}$ & $1^{\circ} 10^{\prime} 13.58^{\prime \prime} \mathrm{E}$ & 13 & $\mathrm{~B}$ & 0.18 & 0.05 & 560 & 5 \\
\hline ERC21b & Sentenac d'Oust & Albian & $42^{\circ} 52^{\prime} 35.49^{\prime \prime} \mathrm{N}$ & $1^{\circ} 10^{\prime} 13.58^{\prime \prime} \mathrm{E}$ & 10 & $\mathrm{~B}$ & 0.21 & 0.06 & 543 & 8 \\
\hline 07LH01 & Lherz & Neocomian & $42^{\circ} 48^{\prime} 13.60^{\prime \prime} \mathrm{N}$ & $1^{\circ} 24^{\prime} 44.87^{\prime \prime} \mathrm{E}$ & 15 & $\mathrm{~B}$ & 0.27 & 0.07 & 520 & 8 \\
\hline 07LH03a & Lherz & Neocomian? & $42^{\circ} 48^{\prime} 50.50^{\prime \prime} \mathrm{N}$ & $1^{\circ} 25^{\prime} 19.86^{\prime \prime} \mathrm{E}$ & 15 & $\mathrm{~B}$ & 0.5 & 0.06 & 428 & 4 \\
\hline ARG14 & Bédeilhac & Triassic gypsum & $42^{\circ} 52^{\prime} 29.59^{\prime \prime} \mathrm{N}$ & $1^{\circ} 34^{\prime} 38.76^{\prime \prime} \mathrm{E}$ & 14 & $\mathrm{~B}$ & 0.65 & 0.01 & 353 & 1 \\
\hline ARG6 & Bédeilhac & Triassic marble & $42^{\circ} 52^{\prime} 30.49^{\prime \prime} \mathrm{N}$ & $1^{\circ} 34^{\prime} 36.71^{\prime \prime} \mathrm{E}$ & 10 & B & 0.16 & 0.08 & 568 & 9 \\
\hline $\mathrm{LC} 05 \mathrm{f}$ & Lherz & Undefined Brecciated pelite & $42^{\circ} 48^{\prime} 49.49^{\prime \prime} \mathrm{N}$ & $1^{\circ} 22^{\prime} 11.90^{\prime \prime} \mathrm{E}$ & 12 & $\mathrm{~B}$ & 0.52 & 0.05 & 412 & 7 \\
\hline LHZ50 & Lherz & Liassic & $42^{\circ} 48^{\prime} 59.05^{\prime \prime} \mathrm{N}$ & $1^{\circ} 21^{\prime} 44.55^{\prime \prime} \mathrm{E}$ & 13 & $\mathrm{~B}$ & 0.5 & 0.06 & 421 & 7 \\
\hline LHZ63 & Lherz & Berriasian-Hauterivian? & $42^{\circ} 48^{\prime} 43.47^{\prime \prime} \mathrm{N}$ & $1^{\circ} 22^{\prime} 15.74^{\prime \prime} \mathrm{E}$ & 10 & $\mathrm{~B}$ & 0.17 & 0.04 & 564 & 6 \\
\hline LHZ65a & Lherz & Triassic & $42^{\circ} 47^{\prime} 51.93^{\prime \prime} \mathrm{N}$ & $1^{\circ} 22^{\prime} 40.30^{\prime \prime} \mathrm{E}$ & 8 & $\mathrm{~B}$ & 0.19 & 0.07 & 556 & 11 \\
\hline LHZ66 & Lherz & Jurassic & $42^{\circ} 47^{\prime} 42.11^{\prime \prime} \mathrm{N}$ & $1^{\circ} 22^{\prime} 53.26^{\prime \prime} \mathrm{E}$ & 10 & $\mathrm{~B}$ & 0.22 & 0.08 & 545 & 11 \\
\hline LHZ67 & Lherz & Jurassic & $42^{\circ} 47^{\prime} 39.03^{\prime \prime} \mathrm{N}$ & $1^{\circ} 23^{\prime} 3.84^{\prime \prime} \mathrm{E}$ & 8 & $\mathrm{~B}$ & 0.25 & 0.05 & 531 & 7 \\
\hline LHZ68 & Lherz & Jurassic & $42^{\circ} 47^{\prime} 53.78^{\prime \prime} \mathrm{N}$ & $1^{\circ} 22^{\prime} 41.49^{\prime \prime} \mathrm{E}$ & 11 & $\mathrm{~B}$ & 0.27 & 0.1 & 523 & 13 \\
\hline LHZ69 & Lherz & Jurassic & $42^{\circ} 47^{\prime} 42.87^{\prime \prime} \mathrm{N}$ & $1^{\circ} 22^{\prime} 25.70^{\prime \prime} \mathrm{E}$ & 6 & $\mathrm{~B}$ & 0.45 & 0.06 & 437 & 8 \\
\hline LHZ75 & Lherz & Berriasian-Hauterivian & $42^{\circ} 48^{\prime} 14.16^{\prime \prime} \mathrm{N}$ & $1^{\circ} 24^{\prime} 44.71^{\prime \prime} \mathrm{E}$ & 11 & $\mathrm{~B}$ & 0.22 & 0.07 & 541 & 9 \\
\hline LHZ76 & Lherz & Berriasian-Hauterivian & $42^{\circ} 48^{\prime} 13.94^{\prime \prime} \mathrm{N}$ & $1^{\circ} 24^{\prime} 44.64^{\prime \prime} \mathrm{E}$ & 10 & $\mathrm{~B}$ & 0.22 & 0.08 & 543 & 11 \\
\hline LHZ77 & Lherz & Berriasian-Hauterivian & $42^{\circ} 48^{\prime} 13.27^{\prime \prime} \mathrm{N}$ & $1^{\circ} 24^{\prime} 45.12^{\prime \prime} \mathrm{E}$ & 12 & $\mathrm{~B}$ & 0.23 & 0.05 & 540 & 6 \\
\hline LHZ78 & Lherz & Jurassic & $42^{\circ} 47^{\prime} 28.11^{\prime \prime} \mathrm{N}$ & $1^{\circ} 24^{\prime} 23.71^{\prime \prime} \mathrm{E}$ & 12 & $\mathrm{~B}$ & 0.23 & 0.07 & 538 & 10 \\
\hline LHZ79 & Lherz & Jurassic & $42^{\circ} 47^{\prime} 48.52^{\prime \prime} \mathrm{N}$ & $1^{\circ} 24^{\prime} 17.17^{\prime \prime} \mathrm{E}$ & 11 & $\mathrm{~B}$ & 0.19 & 0.13 & 556 & 17 \\
\hline LHZ80 & Lherz & Liassic & $42^{\circ} 47^{\prime} 54.42^{\prime \prime} \mathrm{N}$ & $1^{\circ} 24^{\prime} 17.08^{\prime \prime} \mathrm{E}$ & 13 & $\mathrm{~B}$ & 0.18 & 0.65 & 560 & 8 \\
\hline LHZ82 & Lherz & Jurassic & $42^{\circ} 47^{\prime} 21.07^{\prime \prime} \mathrm{N}$ & $1^{\circ} 24^{\prime} 24.75^{\prime \prime} \mathrm{E}$ & 10 & $\mathrm{~B}$ & 0.23 & 0.05 & 537 & 6 \\
\hline LHZ83 & Lherz & Liassic & $42^{\circ} 46^{\prime} 56.23^{\prime \prime} \mathrm{N}$ & $1^{\circ} 24^{\prime} 17.22^{\prime \prime} \mathrm{E}$ & 11 & $\mathrm{~B}$ & 0.23 & 0.09 & 540 & 13 \\
\hline LHZ85 & Lherz & Turonian-Senonian & $42^{\circ} 46^{\prime} 53.41^{\prime \prime} \mathrm{N}$ & $1^{\circ} 23^{\prime} 43.51^{\prime \prime} \mathrm{E}$ & 7 & $\mathrm{~L}$ & 0.63 & 0.01 & 321 & 5 \\
\hline LHZ86 & Lherz & Devonian & $42^{\circ} 46^{\prime} 53.13^{\prime \prime} \mathrm{N}$ & $1^{\circ} 23^{\prime} 43.58^{\prime \prime} \mathrm{E}$ & 9 & $\mathrm{~B}$ & 0.2 & 0.05 & 551 & 8 \\
\hline LHZ87 & Lherz & Berriasian-Hauterivian? & $42^{\circ} 48^{\prime} 45.50^{\prime \prime} \mathrm{N}$ & $1^{\circ} 22^{\prime} 15.00^{\prime \prime} \mathrm{E}$ & 9 & $\mathrm{~B}$ & 0.19 & 0.07 & 557 & 10 \\
\hline LHZ90 & Lherz & Liassic & $42^{\circ} 47^{\prime} 15.70^{\prime \prime} \mathrm{N}$ & $1^{\circ} 23^{\prime} 3.90^{\prime \prime} \mathrm{E}$ & 14 & $\mathrm{~B}$ & 0.03 & 0.03 & 626 & 4 \\
\hline LHZ91 & Lherz & Turonian-Senonian & $42^{\circ} 47^{\prime} 13.83^{\prime \prime} \mathrm{N}$ & $1^{\circ} 21^{\prime} 9.54^{\prime \prime} \mathrm{E}$ & 15 & $\mathrm{~L}$ & 0.65 & 0.02 & 343 & 6 \\
\hline LHZ92 & Lherz & Devonian & $42^{\circ} 46^{\prime} 50.10^{\prime \prime} \mathrm{N}$ & $1^{\circ} 22^{\prime} 26.50^{\prime \prime} \mathrm{E}$ & 18 & $\mathrm{~B}$ & 0.31 & 0.06 & 505 & 6 \\
\hline LHZ93 & Lherz & Turonian-Senonian & $42^{\circ} 46^{\prime} 26.50^{\prime \prime} \mathrm{N}$ & $1^{\circ} 27^{\prime} 18.50^{\prime \prime} \mathrm{E}$ & 16 & $\mathrm{~L}$ & 0.65 & 0.02 & 336 & 5 \\
\hline LHZ94 & Lherz & Turonian-Senonian & $42^{\circ} 46^{\prime} 40.73^{\prime \prime} \mathrm{N}$ & $1^{\circ} 26^{\prime} 22.06^{\prime \prime} \mathrm{E}$ & 15 & $\mathrm{~L}$ & 0.67 & 0.03 & 365 & 9 \\
\hline LHZ95 & Lherz & Liassic & $42^{\circ} 46^{\prime} 50.60^{\prime \prime} \mathrm{N}$ & $1^{\circ} 25^{\prime} 49.70^{\prime \prime} \mathrm{E}$ & 15 & $\mathrm{~B}$ & 0.18 & 0.06 & 559 & 7 \\
\hline LHZ96 & Lherz & Liassic & $42^{\circ} 46^{\prime} 54.60^{\prime \prime} \mathrm{N}$ & $1^{\circ} 25^{\prime} 3.00^{\prime \prime} \mathrm{E}$ & 16 & $\mathrm{~B}$ & 0.19 & 0.08 & 557 & 7 \\
\hline LHZ98 & Lherz & Turonian-Senonian & $42^{\circ} 46^{\prime} 30.60^{\prime \prime} \mathrm{N}$ & $1^{\circ} 27^{\prime} 48.70^{\prime \prime} \mathrm{E}$ & 13 & $\mathrm{~L}$ & 0.66 & 0.04 & 341 & 6 \\
\hline SUC3 & Vicdessos & Turonian-Senonian & $42^{\circ} 46^{\prime} 21.19^{\prime \prime} \mathrm{N}$ & $1^{\circ} 29^{\prime} 31.87^{\prime \prime} \mathrm{E}$ & 12 & $\mathrm{~B}$ & 0.65 & 0.03 & 353 & 4 \\
\hline $\mathrm{SX} 2$ & Lherz & Black metapelite (Liassic?) & $42^{\circ} 46^{\prime} 56.11^{\prime \prime} \mathrm{N}$ & $1^{\circ} 23^{\prime} 59.86^{\prime \prime} \mathrm{E}$ & 8 & $\mathrm{~B}$ & 0.15 & 0.07 & 576 & 10 \\
\hline MP10 & Moncaup & Liassic & $42^{\circ} 58^{\prime} 53.64^{\prime \prime} \mathrm{N}$ & $0^{\circ} 42^{\prime} 38.04^{\prime \prime} \mathrm{E}$ & 4 & $\mathrm{~L}$ & 0.56 & 0.07 & 235 & 42 \\
\hline MP12 & Moncaup & Liassic & $42^{\circ} 59^{\prime} 30.10^{\prime \prime} \mathrm{N}$ & $0^{\circ} 43^{\prime} 19.80^{\prime \prime} \mathrm{E}$ & 12 & $\mathrm{~L}$ & 0.64 & 0.03 & 333 & 8 \\
\hline MP13 & Moncaup & Liassic & $42^{\circ} 59^{\prime} 31.40^{\prime \prime} \mathrm{N}$ & $0^{\circ} 43^{\prime} 13.20^{\prime \prime} \mathrm{E}$ & 17 & $\mathrm{~L}$ & 0.63 & 0.02 & 322 & 5 \\
\hline MP14 & Moncaup & Liassic & $42^{\circ} 59^{\prime} 25.80^{\prime \prime} \mathrm{N}$ & $0^{\circ} 43^{\prime} 7.70^{\prime \prime} \mathrm{E}$ & 10 & $\mathrm{~B}$ & 0.64 & 0.02 & 358 & 2 \\
\hline MP16 & Moncaup & Barremian & $42^{\circ} 58^{\prime} 3.50^{\prime \prime} \mathrm{N}$ & $0^{\circ} 42^{\prime} 38.40^{\prime \prime} \mathrm{E}$ & 10 & $\mathrm{~L}$ & 0.6 & 0.03 & 303 & 7 \\
\hline MP17 & Moncaup & Jurassic & $42^{\circ} 57^{\prime} 50.30^{\prime \prime} \mathrm{N}$ & $0^{\circ} 43^{\prime} 36.20^{\prime \prime} \mathrm{E}$ & 17 & B & 0.12 & 0.12 & 604 & 8 \\
\hline MP18 & Moncaup & Jurassic & $42^{\circ} 57^{\prime} 49.20^{\prime \prime} \mathrm{N}$ & $0^{\circ} 43^{\prime} 35.70^{\prime \prime} \mathrm{E}$ & 24 & $\mathrm{~B}$ & 0.18 & 0.15 & 587 & 10 \\
\hline MP21 & Moncaup & Gargasian to early Albian & $42^{\circ} 57^{\prime} 36.00^{\prime \prime} \mathrm{N}$ & $0^{\circ} 43^{\prime} 12.00^{\prime \prime} \mathrm{E}$ & 18 & $\mathrm{~L}$ & 0.6 & 0.07 & 301 & 5 \\
\hline MP22 & Moncaup & Urgonian & $42^{\circ} 57^{\prime} 47.20^{\prime \prime} \mathrm{N}$ & $0^{\circ} 42^{\prime} 57.90^{\prime \prime} \mathrm{E}$ & 20 & $\mathrm{~L}$ & 0.63 & 0.02 & 320 & 5 \\
\hline MP23 & Moncaup & Late Jurassic & $42^{\circ} 58^{\prime} 10.40^{\prime \prime} \mathrm{N}$ & $0^{\circ} 42^{\prime} 27.40^{\prime \prime} \mathrm{E}$ & 17 & $\mathrm{~L}$ & 0.61 & 0.03 & 289 & 9 \\
\hline MP26 & Moncaup & Liassic & $42^{\circ} 56^{\prime} 52.50^{\prime \prime} \mathrm{N}$ & $0^{\circ} 44^{\prime} 12.80^{\prime \prime} \mathrm{E}$ & 16 & $\mathrm{~B}$ & 0.48 & 0.14 & 406 & 5 \\
\hline MP27 total & Moncaup & Jurassic (Kimmeridgian) & $42^{\circ} 57^{\prime} 23.50^{\prime \prime} \mathrm{N}$ & $0^{\circ} 44^{\prime} 25.60^{\prime \prime} \mathrm{E}$ & 23 & $\mathrm{~B}$ & 0.47 & 0.1 & 425 & 6 \\
\hline MP27d cl bl & Moncaup & clast in Kimmeridgian breccia & $42^{\circ} 57^{\prime} 23.50^{\prime \prime} \mathrm{N}$ & $0^{\circ} 44^{\prime} 25.60^{\prime \prime} \mathrm{E}$ & 4 & B & 0.52 & 0.03 & 410 & 6 \\
\hline MP27e cl n & Moncaup & clast in Kimmeridgian breccia & $42^{\circ} 57^{\prime} 23.50^{\prime \prime} \mathrm{N}$ & $0^{\circ} 44^{\prime} 25.60^{\prime \prime} \mathrm{E}$ & 13 & $\mathrm{~B}$ & 0.47 & 0.08 & 431 & 10 \\
\hline MP27e cl ool & Moncaup & clast in Kimmeridgian breccia & $42^{\circ} 57^{\prime} 23.50^{\prime \prime} \mathrm{N}$ & $0^{\circ} 44^{\prime} 25.60^{\prime \prime} \mathrm{E}$ & 6 & $\mathrm{~B}$ & 0.49 & 0.05 & 423 & 9 \\
\hline
\end{tabular}


Table 1. Continued.

\begin{tabular}{|c|c|c|c|c|c|c|c|c|c|c|}
\hline \multirow{2}{*}{$\begin{array}{l}\text { Sample } \\
\text { MP28 }\end{array}$} & \multirow{2}{*}{$\begin{array}{l}\text { Location } \\
\text { Moncaup }\end{array}$} & \multirow{2}{*}{$\begin{array}{l}\text { Lithology } \\
\text { Jurassic }\end{array}$} & \multicolumn{2}{|c|}{ Position } & \multirow{2}{*}{$\begin{array}{l}\mathrm{Nb} \\
\text { Sprectra } \\
17\end{array}$} & \multirow{2}{*}{$\begin{array}{l}\text { Method } \\
\text { B }\end{array}$} & \multirow{2}{*}{$\begin{array}{l}\begin{array}{l}\text { Raman } \\
\text { parameter }\end{array} \\
0.44\end{array}$} & \multirow{2}{*}{$\begin{array}{l}\text { SD } \\
0.09\end{array}$} & \multirow{2}{*}{$\begin{array}{l}\mathrm{T} \\
\left({ }^{\circ} \mathrm{C}\right)\end{array}$} & \multirow{2}{*}{$\begin{array}{l}\mathrm{SE} \\
\left({ }^{\circ} \mathrm{C}\right)\end{array}$} \\
\hline & & & $42^{\circ} 57^{\prime} 20.00^{\prime \prime} \mathrm{N}$ & $0^{\circ} 44^{\prime} 20.60^{\prime \prime} \mathrm{E}$ & & & & & & \\
\hline MP29 & Moncaup & Jurassic & $42^{\circ} 57^{\prime} 15.63^{\prime \prime} \mathrm{N}$ & $0^{\circ} 44^{\prime} 17.94^{\prime \prime} \mathrm{E}$ & 20 & $\mathrm{~B}$ & 0.42 & 0.09 & 448 & 9 \\
\hline MP30 & Moncaup & Jurassic & $42^{\circ} 57^{\prime} 12.20^{\prime \prime} \mathrm{N}$ & $0^{\circ} 44^{\prime} 17.90^{\prime \prime} \mathrm{E}$ & 15 & $\mathrm{~B}$ & 0.47 & 0.08 & 427 & 7 \\
\hline MP31 & Moncaup & Jurassic & $42^{\circ} 57^{\prime} 8.90^{\prime \prime} \mathrm{N}$ & $0^{\circ} 44^{\prime} 13.00^{\prime \prime} \mathrm{E}$ & 17 & B & 0.59 & 0.03 & 380 & 4 \\
\hline MP34 & Moncaup & Jurassic & $42^{\circ} 57^{\prime} 0.50^{\prime \prime} \mathrm{N}$ & $0^{\circ} 44^{\prime} 18.80^{\prime \prime} \mathrm{E}$ & 2 & $\mathrm{~B}$ & 0.58 & 0.01 & 383 & 5 \\
\hline MP35 & Moncaup & Jurassic & $42^{\circ} 57^{\prime} 0.50^{\prime \prime} \mathrm{N}$ & $0^{\circ} 44^{\prime} 18.80^{\prime \prime} \mathrm{E}$ & 24 & $\mathrm{~L}$ & 0.63 & 0.04 & 319 & 9 \\
\hline MP36 & Moncaup & Jurassic & $42^{\circ} 57^{\prime} 00.50^{\prime \prime} \mathrm{N}$ & $0^{\circ} 44^{\prime} 18.80^{\prime \prime} \mathrm{E}$ & 2 & $\mathrm{~L}$ & 0.64 & 0.01 & 330 & 9 \\
\hline MP37a & Moncaup & Berriasien & $42^{\circ} 57^{\prime} 04.60^{\prime \prime} \mathrm{N}$ & $0^{\circ} 43^{\prime} 54.10^{\prime \prime} \mathrm{E}$ & 18 & $\mathrm{~L}$ & 0.64 & 0.01 & 327 & 4 \\
\hline MP38a & Moncaup & Paleozoic? Liassic? & $42^{\circ} 59^{\prime} 07.73^{\prime \prime} \mathrm{N}$ & $0^{\circ} 44^{\prime} 20.47^{\prime \prime} \mathrm{E}$ & 19 & $\mathrm{~B}$ & 0.12 & 0.09 & 594 & 5 \\
\hline 11MP39 & Moncaup & Triassic? & $42^{\circ} 59^{\prime} 09.07^{\prime \prime} \mathrm{N}$ & $0^{\circ} 44^{\prime} 21.21^{\prime \prime} \mathrm{E}$ & 16 & $\mathrm{~B}$ & 0.19 & 0.08 & 555 & 9 \\
\hline $11 \mathrm{MP} 40$ & Moncaup & Triassic? & $42^{\circ} 59^{\prime} 08.85^{\prime \prime} \mathrm{N}$ & $0^{\circ} 44^{\prime} 21.08^{\prime \prime} \mathrm{E}$ & 10 & $\mathrm{~B}$ & 0.18 & 0.07 & 559 & 10 \\
\hline SAR 34 & Saraillé & Albian-Cenomanian & $0^{\circ} 42^{\prime} 38.04^{\prime \prime} \mathrm{E}$ & $0^{\circ} 37^{\prime} 22.85^{\prime \prime} \mathrm{W}$ & 10 & $\mathrm{~B}$ & 0.66 & 0.02 & 346 & 3 \\
\hline SAR5 & Saraillé & Urgonian & $43^{\circ} 03^{\prime} 9.89^{\prime \prime} \mathrm{N}$ & $0^{\circ} 38^{\prime} 24.90^{\prime \prime} \mathrm{W}$ & 7 & $\mathrm{~L}$ & 0.61 & 0.03 & 297 & 14 \\
\hline SAR7 & Saraillé & Urgonian & $43^{\circ} 03^{\prime} 11.44^{\prime \prime} \mathrm{N}$ & $0^{\circ} 38^{\prime} 14.71^{\prime \prime} \mathrm{W}$ & 15 & $\mathrm{~L}$ & 0.64 & 0.02 & 328 & 7 \\
\hline URD11 & Urdach & Cenomanian & $43^{\circ} 07^{\prime} 07.60^{\prime \prime} \mathrm{N}$ & $0^{\circ} 39^{\prime} 46.12^{\prime \prime} \mathrm{W}$ & 8 & $\mathrm{~L}$ & 0.55 & 0.02 & 215 & 3 \\
\hline URD14 & Urdach & Cenomanian & $43^{\circ} 07^{\prime} 07.60^{\prime \prime} \mathrm{N}$ & $0^{\circ} 39^{\prime} 46.12^{\prime \prime} \mathrm{W}$ & 13 & $\mathrm{~L}$ & 0.57 & 0 & 233 & 1 \\
\hline 10AG2B & Agly & Liassic & $42^{\circ} 45^{\prime} 29.48^{\prime \prime} \mathrm{N}$ & $2^{\circ} 45^{\prime} 27.76^{\prime \prime} \mathrm{E}$ & 14 & $\mathrm{~B}$ & 0.68 & 0.03 & 337 & 4 \\
\hline 10AG01B & Agly & Jurassic & $42^{\circ} 45^{\prime} 14.10^{\prime \prime} \mathrm{N}$ & $2^{\circ} 46^{\prime} 31.39^{\prime \prime} \mathrm{E}$ & 15 & $\mathrm{~B}$ & 0.64 & 0.01 & 356 & 2 \\
\hline $10 \mathrm{AG} 04 \mathrm{~A}$ & Agly & Triassic & $42^{\circ} 45^{\prime} 04.46^{\prime \prime} \mathrm{N}$ & $2^{\circ} 46^{\prime} 5.73^{\prime \prime} \mathrm{E}$ & 12 & $\mathrm{~B}$ & 0.57 & 0.05 & 387 & 6 \\
\hline $10 \mathrm{AG} 03$ & Agly & Silurian & $42^{\circ} 44^{\prime} 53.24^{\prime \prime} \mathrm{N}$ & $2^{\circ} 46^{\prime} 14.76^{\prime \prime} \mathrm{E}$ & 14 & $\mathrm{~B}$ & 0.65 & 0.02 & 351 & 3 \\
\hline 10AG4B & Agly & & $42^{\circ} 45^{\prime} 04.68^{\prime \prime} \mathrm{N}$ & $2^{\circ} 46^{\prime} 3.03^{\prime \prime} \mathrm{E}$ & 13 & $\mathrm{~B}$ & 0.33 & 0.06 & 494 & 7 \\
\hline 12 PB 02 & Abarratia & Turonian? & $43^{\circ} 20^{\prime} 56.18^{\prime \prime} \mathrm{N}$ & $1^{\circ} 14^{\prime} 16.30^{\prime \prime} \mathrm{W}$ & 20 & $\mathrm{~L}$ & 0.5 & 0.08 & $<200$ & 21 \\
\hline 12 PB 03 & col d'Ibarburia & Aptian-Albian "Marnes à Spicules" & $43^{\circ} 05^{\prime} 41.00^{\prime \prime} \mathrm{N}$ & $1^{\circ} 0^{\prime} 54.00^{\prime \prime} \mathrm{W}$ & 19 & $\mathrm{~L}$ & 0.57 & 0.02 & 249 & 2 \\
\hline 12 PB 05 & Arhansus & Albian-Cenomanian & $43^{\circ} 04^{\prime} 20.70^{\prime \prime} \mathrm{N}$ & $1^{\circ} 1^{\prime} 11.60^{\prime \prime} \mathrm{W}$ & 17 & $\mathrm{~L}$ & 0.55 & 0.01 & 224 & 3 \\
\hline 12 PB 06 & Arhansus & Albian-Cenomanian & $43^{\circ} 04^{\prime} 17.80^{\prime \prime} \mathrm{N}$ & $1^{\circ} 1^{\prime} 19.80^{\prime \prime} \mathrm{W}$ & 19 & $\mathrm{~L}$ & 0.56 & 0.02 & 235 & 4 \\
\hline 12 PB 07 & Barlanès & Albian-Cenomanian & $43^{\circ} 02^{\prime} 43.00^{\prime \prime} \mathrm{N}$ & $0^{\circ} 47^{\prime} 21.40^{\prime \prime} \mathrm{W}$ & 16 & $\mathrm{~L}$ & 0.49 & 0.05 & 156 & 10 \\
\hline 12 PB 08 & Barlanès & Liassic & $43^{\circ} 03^{\prime} 23.30^{\prime \prime} \mathrm{N}$ & $0^{\circ} 47^{\prime} 40.40^{\prime \prime} \mathrm{W}$ & 11 & $\mathrm{~L}$ & 0.61 & 0.01 & 288 & 4 \\
\hline 12 PB 09 & Barlanès & Albian-Cenomanian & $43^{\circ} 04^{\prime} 55.10^{\prime \prime} \mathrm{N}$ & $0^{\circ} 48^{\prime} 3.70^{\prime \prime} \mathrm{W}$ & 2 & $\mathrm{~L}$ & 0.67 & 0.06 & 366 & 56 \\
\hline 12 PB 10 & Roquiague & Albian-Cenomanian & $43^{\circ} 06^{\prime} 18.00^{\prime \prime} \mathrm{N}$ & $0^{\circ} 51^{\prime} 15.40^{\prime \prime} \mathrm{W}$ & 18 & $\mathrm{~L}$ & 0.59 & 0.04 & 255 & 5 \\
\hline 12 PB 11 & Roquiague & Early Cenomanian & $43^{\circ} 07^{\prime} 31.50^{\prime \prime} \mathrm{N}$ & $0^{\circ} 48^{\prime} 32.00^{\prime \prime} \mathrm{W}$ & 20 & $\mathrm{~L}$ & 0.64 & 0.02 & 331 & 4 \\
\hline 12 PB 12 & Roquiague & Early Cenomanian & $43^{\circ} 10^{\prime} 04.30^{\prime \prime} \mathrm{N}$ & $0^{\circ} 46^{\prime} 25.60^{\prime \prime} \mathrm{W}$ & 19 & $\mathrm{~L}$ & 0.64 & 0.02 & 330 & 5 \\
\hline 12 PB 13 & Roquiague & Late Cenomanian & $43^{\circ} 08^{\prime} 37.80^{\prime \prime} \mathrm{N}$ & $0^{\circ} 48^{\prime} 33.62^{\prime \prime} \mathrm{W}$ & 19 & $\mathrm{~L}$ & 0.57 & 0.01 & 247 & 2 \\
\hline 12 PB 15 & Roquiague & Cenomanian-Turonian & $43^{\circ} 12^{\prime} 25.80^{\prime \prime} \mathrm{N}$ & $0^{\circ} 59^{\prime} 38.90^{\prime \prime} \mathrm{W}$ & 20 & $\mathrm{~L}$ & 0.55 & 0.01 & 224 & 3 \\
\hline 12 PB 16 & Roquiague & Aptian-Albian & $43^{\circ} 10^{\prime} 20.10^{\prime \prime} \mathrm{N}$ & $0^{\circ} 57^{\prime} 22.60^{\prime \prime} \mathrm{W}$ & 19 & $\mathrm{~L}$ & 0.61 & 0.01 & 295 & 2 \\
\hline 12 PB 17 & Saraillé & Albian-Cenomanian & $43^{\circ} 02^{\prime} 55.59^{\prime \prime} \mathrm{N}$ & $0^{\circ} 39^{\prime} 56.61^{\prime \prime} \mathrm{W}$ & 19 & $\mathrm{~L}$ & 0.6 & 0.02 & 278 & 5 \\
\hline 12 PB 18 & Saraillé & Albian-Cenomanian & $43^{\circ} 02^{\prime} 25.80^{\prime \prime} \mathrm{N}$ & $0^{\circ} 37^{\prime} 44.70^{\prime \prime} \mathrm{W}$ & 19 & $\mathrm{~L}$ & 0.59 & 0.01 & 263 & 3 \\
\hline 12 PB 19 & Saraillé & Jurassic & $43^{\circ} 03^{\prime} 59.57^{\prime \prime} \mathrm{N}$ & $0^{\circ} 36^{\prime} 07.12^{\prime \prime} \mathrm{W}$ & 20 & $\mathrm{~L}$ & 0.61 & 0.01 & 298 & 2 \\
\hline
\end{tabular}

sanidine standard $(28.03 \pm 0.08 \mathrm{Ma}$; Jourdan and Renne, 2007) as flux monitor. Argon isotopic interferences on $\mathrm{K}$ and $\mathrm{Ca}$ were determined by irradiation of $\mathrm{KF}$ and $\mathrm{CaF}_{2}$ pure salts from which the following correction factors were obtained: $\left({ }^{40} \mathrm{Ar} /{ }^{39} \mathrm{Ar}\right)_{\mathrm{K}}=0.00969 \pm 0.00038,\left({ }^{38} \mathrm{Ar} /{ }^{39} \mathrm{Ar}\right)_{\mathrm{K}}=$ $0.01297 \pm 0.00045,\left({ }^{39} \mathrm{Ar} /{ }^{37} \mathrm{Ar}\right)_{\mathrm{Ca}}=0.0007474 \pm 0.000021$ and $\left({ }^{36} \mathrm{Ar} /{ }^{37} \mathrm{Ar}\right)_{\mathrm{Ca}}=0.000288 \pm 0.000016$. Argon analyses were performed at Géosciences Montpellier (France) with an analytical system that consists of (a) an IR $\mathrm{CO}_{2}$ laser of $100 \mathrm{kHz}$ used at $5-15 \%$ during $60 \mathrm{~s}$; (b) a lense system for beam focusing; (c) a steel chamber, maintained at $10^{-8}-10^{-9}$ bar, with a drilled copper plate;(d) an inlet line for purification of gases including two $\mathrm{Zr}-\mathrm{Al}$ getters; and (e) a MAP215-50 mass spectrometer or an Argus VI Thermo-Fisher multi-collector mass spectrometer. Custommade software controlled the laser intensity, the timing of extraction/purification, the data acquisition and reduction to calculate ages. To measure the argon background within the system, one blank analysis was performed every three sample analyses. The $1 \sigma$ errors reported on plateau, isochron and total gas ages include the error on the irradiation factor $\mathrm{J}$.
Atmospheric ${ }^{40} \mathrm{Ar}$ was estimated using a value of the initial ${ }^{40} \mathrm{Ar}-{ }^{36} \mathrm{Ar}$ of 295.5 .

\subsubsection{Metasediments samples}

Muscovite of samples LHZ120 and FREYCH was extracted from marbles of the Lherz areas (Fig. 8). Sample LHZ107 is a centimeter-sized muscovite crystal extracted from a calcite vein cutting through the Liassic metapelites of Port de Saleix. Muscovites LHZ125 and LHZ152 were sampled in the breccias reworking clasts of Mesozoic marbles exposed near the peridotite bodies of Freychinède (LHZ125) and Etang de Lherz (LHZ152). Similar breccias are described by Clerc et al. (2013). The muscovite grains were extracted from the matrix of the breccias (Fig. 9). Sample 11MP92 is a centimetersized muscovite crystal found in the rim of a mafic intrusion exposed in the Arguenos marble quarry, on the western side of Montégut (Fig. 10a and b). Muscovite StB comes from the Jurassic white marbles of the Rapp quarry, near St Béat. The amphiboles of samples 11MP86 and 11MP87 were obtained from metamorphosed Liassic tuffs (B. Azambre, personal communication, 2014) exposed in fresh cuts along road 


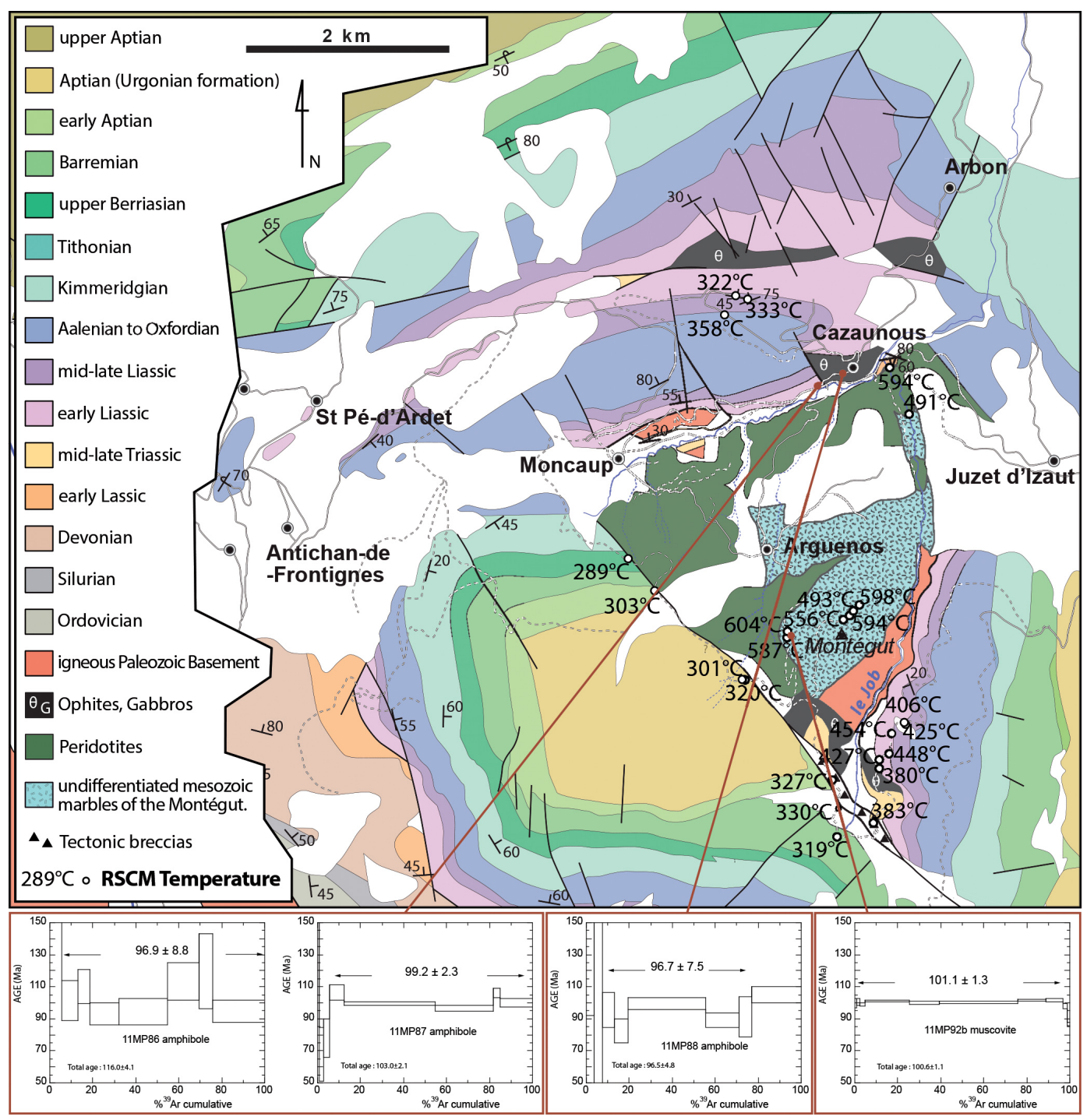

Figure 7. Geological map of the Arguenos-Moncaup area (after Barrère et al., 1984a; Hervouët et al., 1987; Canérot and Debroas, 1988) with Raman temperature values and location of the samples used for ${ }^{40} \mathrm{Ar}-{ }^{39} \mathrm{Ar}$ dating. A clear increase of peak temperature appears in the vicinity of the mantle peridotites.

D39 close to the intersection with roads D618. The tuffs are highly recrystallized, hosting centimeter-long unoriented amphiboles (Fig. 10c, d and e).

\subsubsection{Magmatic rock samples}

Three amphibole samples were extracted from the metadolerites of Freychinède (LHZ146, LHZ148) and from Cazaunous (11MP88). These rocks, generally referred to as ophites, were emplaced during the Triassic (Montigny et al., 1982). The ophites of the NPZ are strongly affected by a post-magmatic transformation responsible for their recrystallization and the neoformation of Al-rich amphibole, clinopyroxene, plagioclase and scapolite under amphibolite facies conditions (Azambre et al., 1987, 1971; Golberg and
Leyreloup, 1990). This recrystallization has been attributed to the Cretaceous thermal event, and the neoformation of amphiboles was dated at $95 \mathrm{Ma}$ by ${ }^{87} \mathrm{Rb}^{87} \mathrm{Sr}$ on amphibole in Lherz (Montigny et al., 1986). The centimeter-long green amphibole of ARI was extracted from gypsum at the Arignac gypsum quarry. TRI is a phlogopite sampled in the hydrothermally altered rim of a pegmatite dyke in the footwall of the Trimouns talc ore deposit. The outcrop is now partly destroyed due to mining excavations.

Two gabbros were sampled in the localities of Port de Saleix (LHZ102, Fig. 8) and Col de Menté (11MP62, out of Fig. 7). At Port de Saleix, the hectometric gabbro body is found on the southernmost part of the Aulus basin, between the NPF and a complex unit containing marble breccias and granulitized metasedimentary rocks including olivine- and 


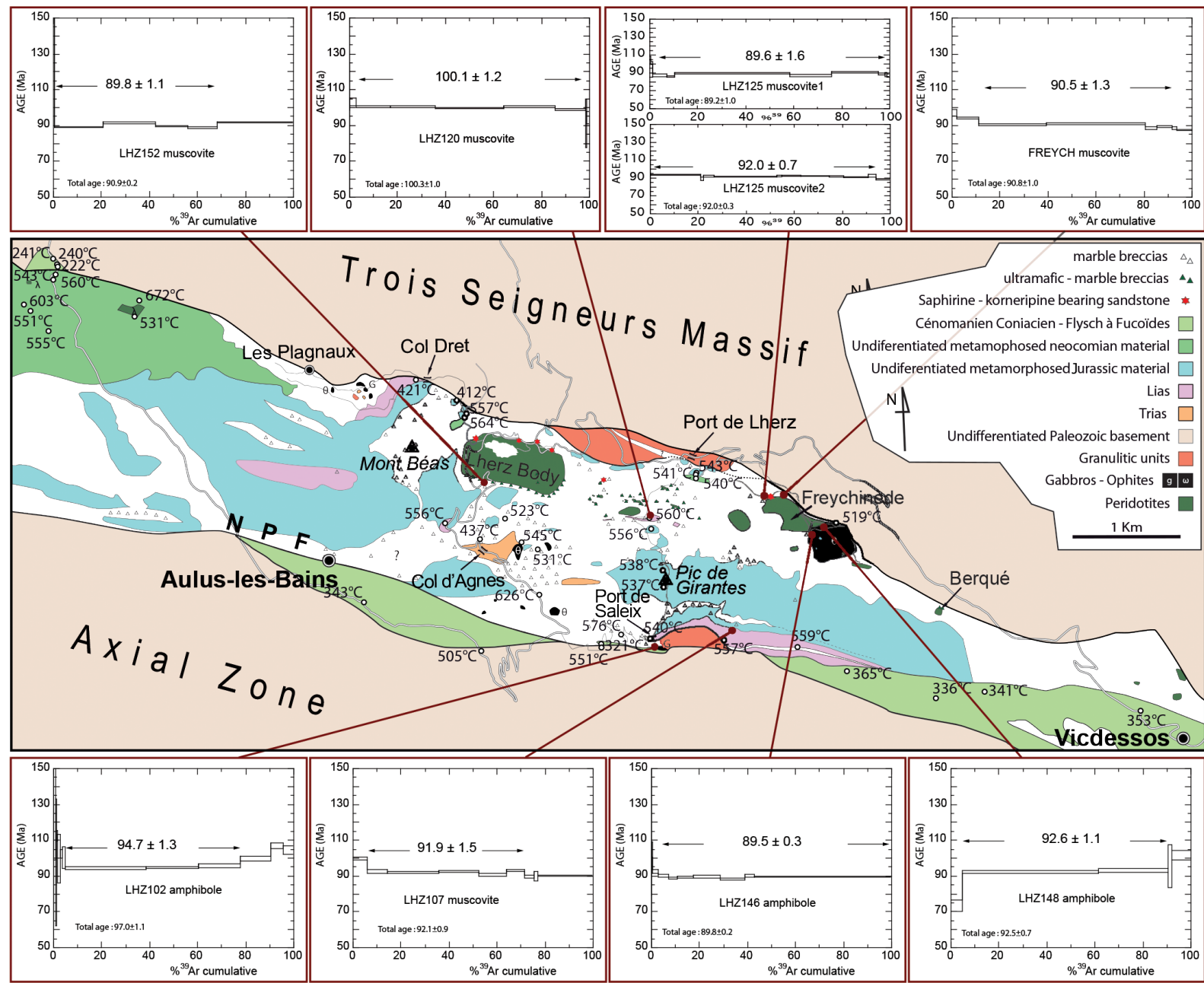

Figure 8. Geological map of the central-eastern part of the Aulus basin, with Raman temperature values and location of samples used for ${ }^{40} \mathrm{Ar}-{ }^{39} \mathrm{Ar}$ dating. No clear thermal trend can be deciphered in the basins, which we interpret as an indication of post-peak-metamorphism disruption of the sedimentary pile.

pyroxene-bearing marbles. At col de Menté, the gabbro constitutes a small intrusion within the Mesozoic marbles. BUZ is a sample of amphibole from a small teschenite dyke close to the village of Buzy (Mauléon basin).

\subsubsection{Results}

The results are presented in Table 2, and relevant areas in Fig. 3 are expanded in Fig. 7 (Moncaup), 8 (Lherz) and 11. In the Lherz area, age spectra are mainly flat for a large percentage of the argon released, with only minor evidence of age scattering due to the presence of tiny inclusions and of weak weathering. Muscovites and amphiboles from six metamorphic rocks provide ages ranging from $89.5 \pm 0.3$ to $92.6 \pm 1.1 \mathrm{Ma}$ (late Cenomanian to Turonian), with the exception of one sample (LHZ120) for which muscovite has a plateau date of 100.1 $\pm 1.2 \mathrm{Ma}$. In the Arguenos-Moncaup areas (Fig. 7), the age spectra of amphiboles are more dis- cordant due the low amount of potassium and probably the contribution of some inclusions. Nevertheless, the cooling ages of all the metamorphic samples are clustering between $101.1 \pm 1.3$ and $96.7 \pm 7.5 \mathrm{Ma}$ (Albian to Cenomanian). Among the metamorphic ages, breccias from the Lherz area gave the youngest ones and are all restricted to the Turonian $(92.0 \pm 0.7,89.8 \pm 1.1$ and $89.6 \pm 1.6 \mathrm{Ma})$.

In both the Arguenos-Moncaup and the Lherz areas, undeformed gabbros yielded amphibole ${ }^{40} \mathrm{Ar}-{ }^{39} \mathrm{Ar}$ ages in the range 94-100 Ma (samples LHZ102, 11MP62), which confirms that the magmatic and metamorphic activities are contemporaneous. We note that this age is younger than the $107-109 \mathrm{Ma}{ }^{40} \mathrm{~K}-{ }^{40} \mathrm{Ar}$ age reported on a gabbro from Les Plagneaux (Fig. 3; Montigny et al., 1986), which may indicate either that magmatism occurred in several pulses between 109 and 94 Ma or that amphiboles from Les Plagneaux have been contaminated by excess argon. However, this age is consistent with the $109.2 \pm 3.5 \mathrm{Ma}$ age obtained on am- 


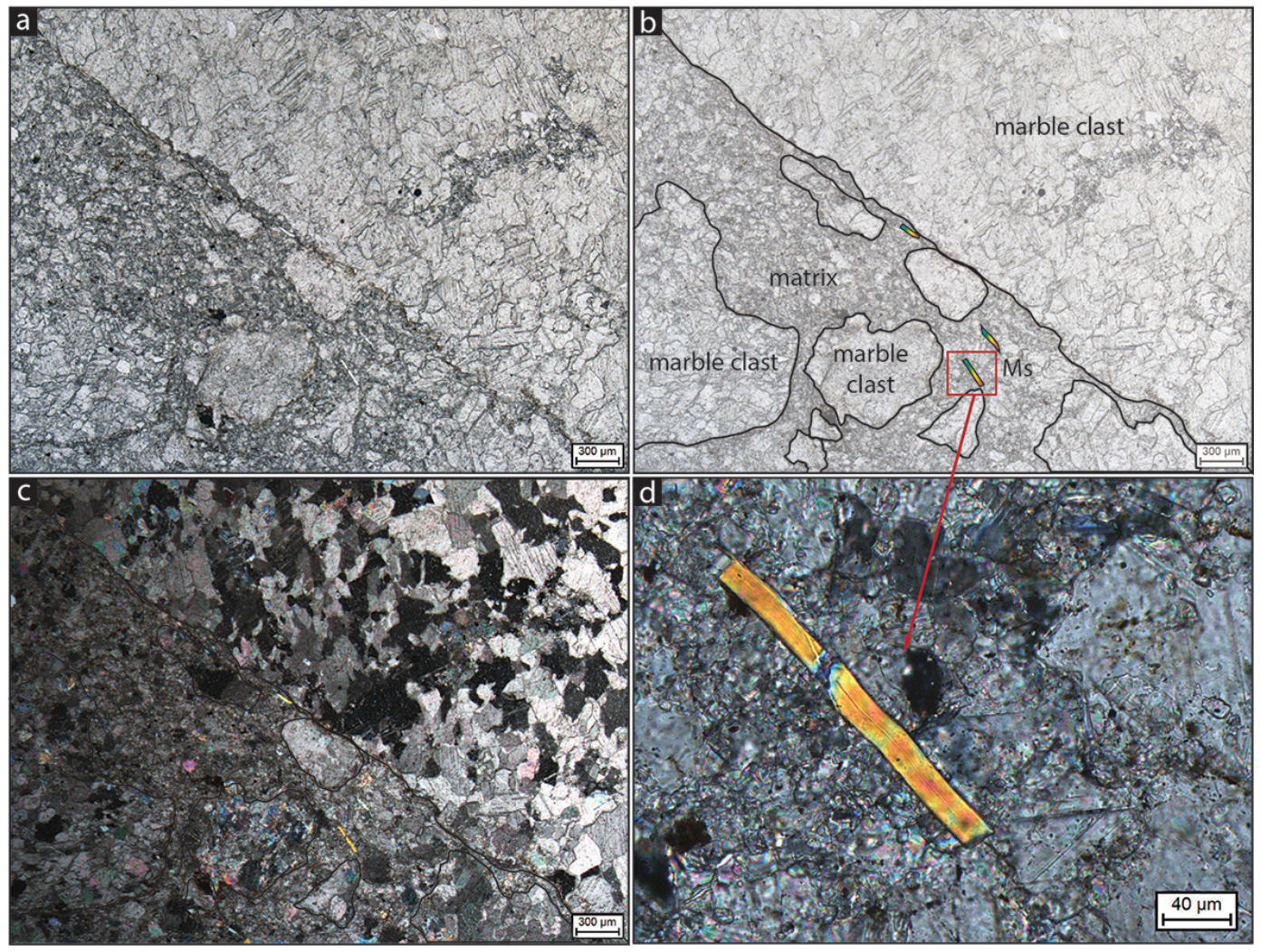

Figure 9. Microscopic view of breccia sample LHZ152. In plane-polarized light (a); redrawn (b); in cross-polarized light (c and d).

phiboles from the Arignac Triassic gypsum (ARI, Fig. 11), which indicates that both metamorphism and magmatism start to affect the Mesozoic cover as soon as the early Albian.

In Tarascon Valley, the $100.3 \pm 1$ Ma age obtained on phlogopite (sample TRI, Fig. 11) from the rim of the Trimouns talc deposit is in agreement with the 112-97 and $99 \mathrm{Ma}$ ages obtained from $\mathrm{U}-\mathrm{Pb}$ dating of xenotime and monazite (Schärer et al., 1999) and constrains the main period of hydrothermal activity to the Albian-Cenomanian transition.

To the west, in Buzy, the teschenite yielded an amphibole ${ }^{40} \mathrm{Ar}-{ }^{39} \mathrm{Ar}$ age of $92.9 \pm 1.3 \mathrm{Ma}$, in good agreement with a previous datum of $93 \pm 4$ Ma by Montigny et al. (1986) which confirms contemporaneity of metamorphism with the emplacement of the previously dated undeformed gabbro bodies in the Cenomanian-lower Turonian.

\subsection{U-Pb dating of albitite veins}

In the Arguenos-Moncaup area, small decametric units of Paleozoic basement resting between the mantle peridotites and the pre-Albian Mesozoic marbles are cross cut by albitite veins (Fig. 12). Ten analyses were performed in 10 different titanite grains present in one thin section (see Supplement for detailed results and analytical procedure). In a Tera Wasserburg diagram (Fig. 13), data plot in a concordant to discordant position and define a lower intercept age of $98.4 \pm 1.1 \mathrm{Ma}(\mathrm{MSWD}=1.4)$ if anchored to a common ${ }^{207} \mathrm{~Pb} /{ }^{206} \mathrm{~Pb}$ value calculated at $100 \mathrm{Ma}$ using a singlestage Stacey and Kramers (1975) value $(98.3 \pm 0.9 \mathrm{Ma}$, MSWD $=1.7 \mathrm{Ma}$ if the regression is free). Therefore we infer that the titanite associated with this albitite crystallized $98 \mathrm{My}$ ago. This age is consistent with the ages of the metamorphic event obtained in this area (Albian to Cenomanian, cf. Sect. 6.1.3).

\section{Discussion}

\subsection{Spatial distribution of the thermal anomaly at the scale of the NPZ}

A first-order zonation of the HT-LP metamorphism appears at the scale of the whole Pyrenean domain. In agreement with the previous results (Choukroune and Séguret 1973; Choukroune 1976; Bernus-Maury 1984; Golberg 1987; Golberg and Leyreloup 1990), we distinguish three main thermal domains along the NPZ (Fig. 2c). From west to east, these are the following:

1. The western domain, corresponding to the Béarn and Pays Basque basins, shows low-grade HT-LP metamorphism with Raman temperatures generally lower than $350^{\circ} \mathrm{C}$. In this part of the belt, the thermal anomaly is 


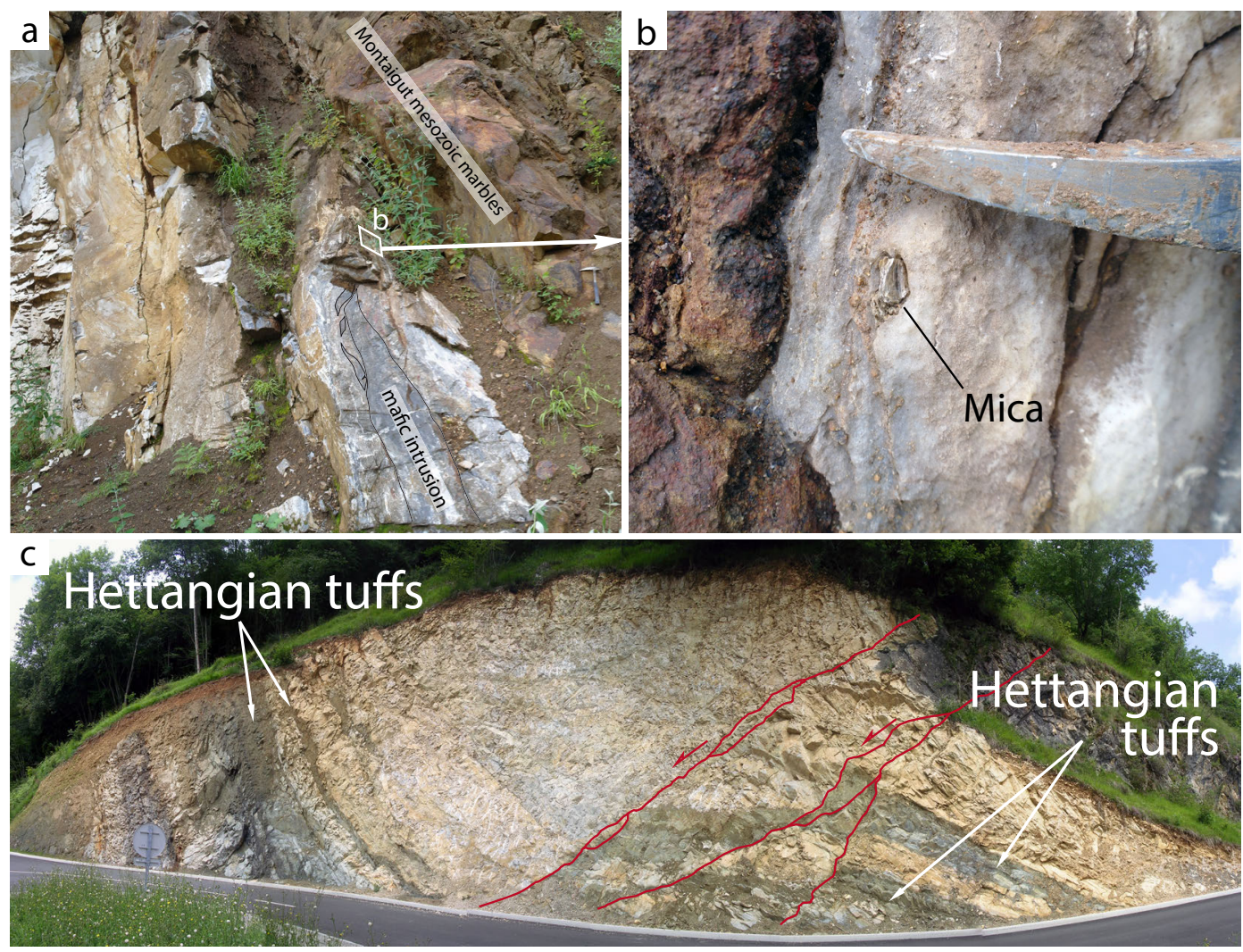

Figure 10. (a) Boudinaged sill in the Montégut marble quarry; (b) detail of (a) showing centimetric muscovite at the contact (sample 11MP92b); (c) two beds of Early Jurassic tuffs intruding marbles at the new outcrop of road D39 (samples 11MP86 and 11MP88).

largely distributed in the prerift metasediments and in the Albian-Cenomanian flyschs, with the highest temperatures found close to exposures of mantle rocks and Paleozoic basement (Saraillé, Roquiague).

2. The central domain - which includes the Ballongue, Barousse and Baronnies basins - displays a higher grade of HT-LP metamorphism, with RSCM temperatures of 300 to $450^{\circ} \mathrm{C}$, locally exceeding $550^{\circ} \mathrm{C}$ close to mantle exposures (Arguenos-Moncaup peridotites body). The temperatures decrease toward the west.

3. The eastern domain - including the Boucheville, Agly, Pays de Sault, and Aulus basins - has the highest grade of HT-LP metamorphism, with Raman temperatures up to $600^{\circ} \mathrm{C}$ and above. In addition to the east-west thermal zonation, a north-south thermal gradient is well observed in this domain. The highest temperatures are found in the southernmost regions (Boucheville and Aulus basins), whereas the northern regions are characterized by a rapid decrease of the HT-LP metamorphic imprint. The isograds are here widely disturbed by later faulting locally outlined by tectonic breccias.

Clerc and Lagabrielle (2014) correlated this thermal zonation across the NPZ to a variable mode of crustal thinning across the pre-Pyrenean paleomargins, suggesting the existence of "hot" margins (eastern domain) in opposition to the cooler western domain. For the authors the three domains can be described as follows:

1. The basins of the cooler western domain are characterized by well-developed coarse clastic formations related to paleoscarps on the border of the troughs, in relation to high-angle faulting and dominant brittle behavior of the continental crust (e.g., the Igountze and Mendibelza breccias; Boirie 1981; Boirie and Souquet 1982; Masini et al., 2014). In contrast, the central part of these basins is a domain of exhumed mantle capped by tectonic lenses of both ultramafic and sialic compositions tectonically underlying the prerift metasediments. Here, thinning of the crust occurred in a ductile mode, but the continental crust does not form large massifs in the center of the basins.

2. The central domain is characterized by basins of smaller dimensions separated by blocks of continental material, the North Pyrenean massifs, whose size globally increases eastward. The basins are frequently triangle- or losange-shaped (Debroas, 1990) with mantle rocks frequently exposed in their central regions. Progressively, toward the east, the continental crust of the North Pyre- 

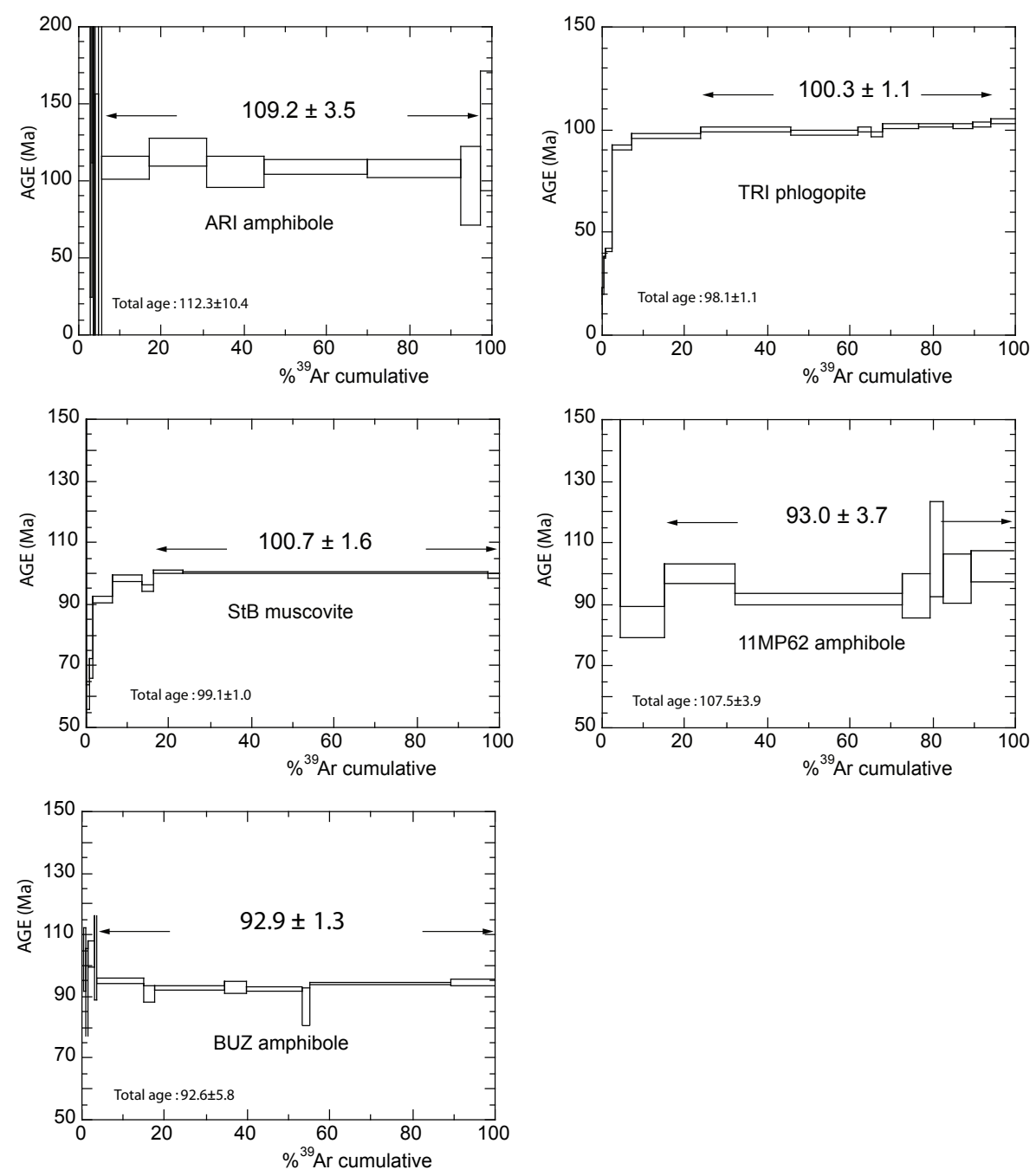

Figure 11. ${ }^{40} \mathrm{Ar}-{ }^{39} \mathrm{Ar}$ step-heating data for the samples ARI, TRI, StB, $11 \mathrm{MP} 62$ and BUZ.

nean massifs is boudinaged and tends to form spindleshaped lenses.

3. The eastern domain is characterized by an intense HT deformation of the Triassic to Albian sedimentary pile and by the boudinage of the continental crust in the distal regions of the paleomargin. The Mesozoic sequence is everywhere severely thinned under a ductile regime, with frequent boudinage and tectonic subtractions of comprehensive portions of the original succession. The Albian-Cenomanian deposits are controlled by a wide wavelength undulation of the top of the lithosphere in relation to the boudinage of the hyper-thinned continental crust.

Several hypotheses are proposed in the following that may explain such a variation of the thermal anomaly along the paleomargin:
- The whole domain underwent similar HT-LP metamorphic evolution, but later tectonic inversion led to underthrusting and burial of the highest-grade rocks in the western part of the NPZ. When considering the general plunge of the Axial Zone toward the west, it is also clear that the eastern part of the belt underwent more exhumation, hence extensively exposing the deepest units that underwent more HT-LP metamorphism. In this case, the western and eastern part of the NPZ could be considered to represent both the shallow (west) and deep (east) processes occurring during the contractional evolution of a single passive margin. However, in that case the prerift material should have undergone temperatures much higher than the 366 and $298^{\circ} \mathrm{C}$ obtained in the Liassic and Jurassic of the Barlanès and Saraillé areas (Table 1; Mauléon basin in Fig. 2).

- By contrast, and in better agreement with the structural variations observed along the paleomargin, we 


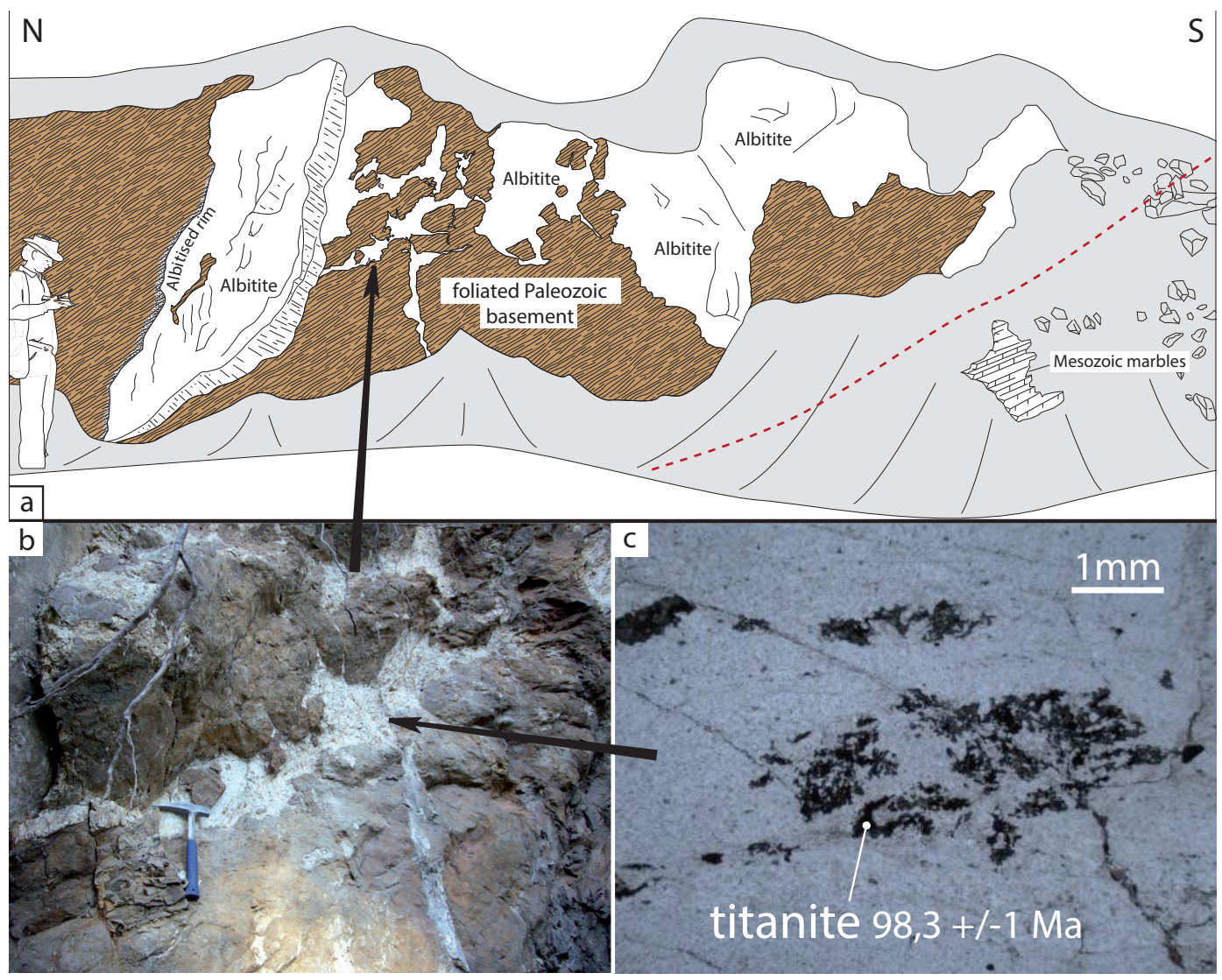

Figure 12. Albitite veins intruding the Paleozoic basement at Arguenos-Moncaup (localized at point 594 near Cazaunous in Fig. 6).

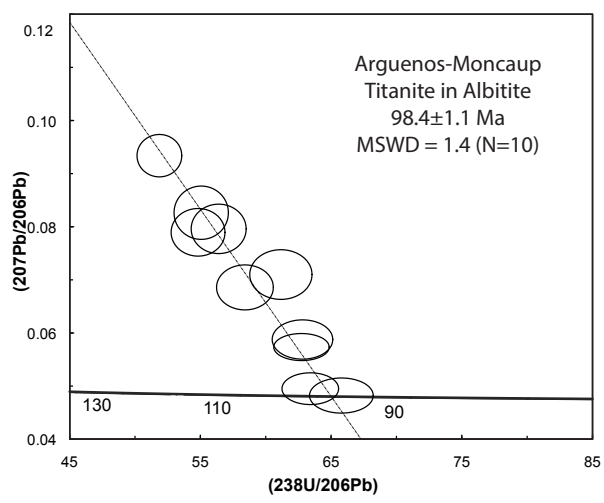

Figure 13. ${ }^{207} \mathrm{~Pb} /{ }^{206} \mathrm{~Pb}-{ }^{238} \mathrm{U} /{ }^{206} \mathrm{~Pb}$ concordia diagram for the 10 spots analyzed in titanite from the albitite veins of AgruenosMoncaup (Fig. 11).

may also consider the thermal zonation of the NPZ as a consequence of a lateral variation of the mode of opening of the basins. Although the kinematic history of the domain is still poorly constrained, approximately $\mathrm{N}-\mathrm{S}$ extension is reported from field observations in the western NPZ (Jammes et al., 2009, 2010; Masini, 2011; Masini et al., 2014), whereas a tran- scurrent to oblique motion is generally reported in the eastern NPZ (Choukroune et Mattauer, 1978; Debroas, 1990; Debroas, 2003; Clerc et al., 2012, Fig. 5). As exemplified in Fig. 6, a rotation pole for the Iberian plate located anywhere in the northeast of the NPZ could lead to differential movement with strike-slip to transtensional extension in the eastern domain and orthogonal to oblique extension in the western domain. Since the deformation is prone to be more localized in a transform system, thermal fluxes are expected to increase with transcurrent motion (Golberg and Leyreloup, 1990; Muffler and White, 1969; McDowell and Elders, 1980, 1983). Furthermore, because the deformation is more localized in the eastern domain than in the western domain, isotherms may have been more spaced in the wider western NPZ than in the narrower eastern NPZ. Regional strain partitioning of a transtensional kinematic into orthogonal extension and transcurrent movement within the future NPZ, the Parentis, Cameros, Le Danois, Basque-Cantabrian and Organya-Pedraforca basins may explain the localization of the HT-LP metamorphism in the basins dominated by transtensive movements (central and eastern NPZ, Nappe des Marbres, Fig. 4), whereas extension 


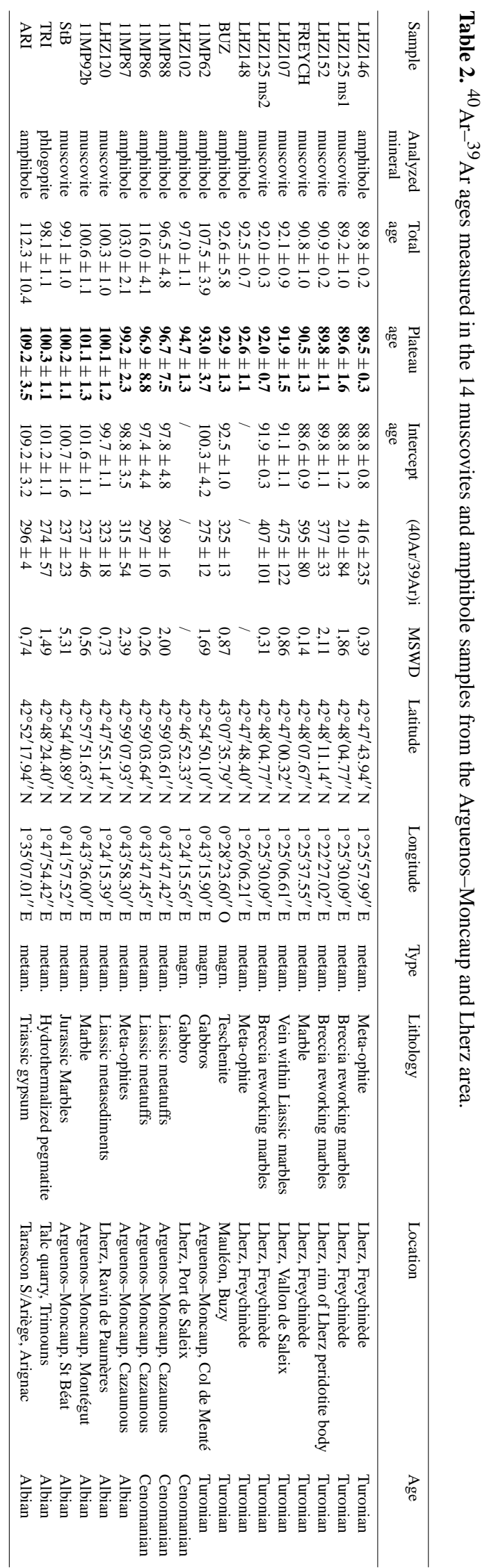

under cooler conditions is registered in the basins undergoing a dominant orthogonal extension (Mauléon basin - Jammes et al., 2009, 2010; Masini et al., 2014). In addition, we can envisage that various modes of opening induced a variety in hydrothermal circulations. If some circulations were externally derived, which is actually not identified in Albian basins using a stable-isotope characterization of veins (Boulvais et al., 2015), they may have produced differences in regional temperature, the coolest temperatures being likely found in the basins with the most efficient cooling circulations (e.g., Souche et al., 2014).

- It is also possible to correlate the intensity of the HT metamorphism to the rising velocity of the mantle peridotites. For the eastern NPZ peridotites, Fabriès et al. (1991) identified a single and rapid decompression and cooling step attributed to their ascent from 50$45 \mathrm{~km}$ depth to the surface. In contrast, for the western NPZ peridotites, Fabriès et al. (1998) identified a slower decompression and cooling from $25-15 \mathrm{~km}$ depth up to the surface (see Fig. 11 in Clerc et al., 2013, for a synthetic exhumation chronology of the NPZ peridotites).

- Finally, the lateral variability of the thermal anomaly along the NPZ might be related to the scissors-shape opening of the extended domain during the Cretaceous (Masson and Miles, 1984; Srivastava et al., 1990; Sibuet and Collette, 1991; Roest and Srivastava, 1991; Sibuet 2004; Jammes et al., 2010).

\subsection{Spatial distribution of the thermal anomaly at the basin scale}

\subsubsection{Reconstructing the initial thermal gradient related to crustal thinning and mantle exhumation: the Arguenos-Moncaup case study}

The spatial distribution of the isograds at the scale of the entire belt shows a first-order relationship between the highest grades of HT metamorphism and the vicinity of deep material exposed in the NPZ (peridotites, granulites, migmatites). Such a relationship was already observed in the eastern part of the NPZ (Golberg and Leyreloup, 1990). Based on their metamorphic analysis in the eastern part of the NPZ, these authors also suggested that these primary relationships were often lost and disturbed by later faulting. Our new data set, extending further west, reveals that the primary relationships between thermal anomalies and basement rocks are better preserved in the central part of the NPZ, and especially in the Arguenos-Moncaup area (Fig. 7), where undisturbed thermal gradients are still recognizable.

The Arguenos-Moncaup ultramafic body is part of a group of peridotite exposures lying around the Milhas massif, in the central Pyrenees (Fig. 2). They are associated with basement rocks, variably brecciated Triassic sediments, ophites and Al- 
bian mafic intrusions. The Arguenos-Moncaup peridotites are overlain in tectonic contact by highly metamorphosed Mesozoic marbles (Debeaux and Thiébaut 1958; Hervouët et al., 1987; Barrère et al., 1984a, b). Although the peridotites have risen to near-surface levels, there is no evidence for sedimentary reworking indicating their exhumation on the basin floor. Indeed, on the basis of their geological setting, it can be deduced that the mantle rocks have remained capped by the Mesozoic marbles together with small slices of continental crust, during their ascent along a detachment fault (Lagabrielle et al., 2010). There, the highest temperatures $\left(500-600^{\circ} \mathrm{C}\right)$ are recorded directly in marbles on top of the peridotite massif, confirming the general trend already suggested from the eastern domain. These Raman temperatures reach values around $400-450^{\circ} \mathrm{C}$ in the metasediments resting over the Paleozoic crustal slice of Job Valley, and they finally decrease to values around $300-350^{\circ} \mathrm{C}$ in the regional background. As suggested by Golberg and Leyreloup (1990), this correlation is the result of the very strong thermal gradient in the locus of the extreme crustal thinning.

At the Pyrenean scale, the original distribution of the thinning-related thermal gradient has not been preserved as well as in the Arguenos-Moncaup area since it has been disrupted by tectonic and sedimentary processes in many places. However, there exist additional remnants of initial relationships, with temperature increasing when approaching the exhumed mantle rocks in some locations (e.g., Saraillé, Roquiague, Montaut, Salvezines).

\subsection{Pre- and post-metamorphic disruption of the sedimentary pile}

\subsubsection{Triassic to Albian metasediments}

An important, well-observed feature of the eastern domain is that the intensity of the peak metamorphism is not correlated with the stratigraphic age. High temperatures are found in Triassic or Albian sediments as well. Also, in the Aulus basin (Fig. 8) peak temperatures of 420 to $600^{\circ} \mathrm{C}$ have been measured in Neocomian material as well as in Jurassic or Triassic material; e.g., one of the coolest temperatures of the Aulus basins has been obtained in the Liassic fossiliferous marbles of the Col Dret. This apparent chaotic disposition of the isotherms within the Triassic to Albian sedimentary pile can be explained by a combination of several mechanisms:

- It can be the consequence of the propagation of the thermal anomaly by magmas or fluid circulations, as already proposed by Dauteuil and Ricou (1989). Fluids circulating through the sedimentary pile could be responsible for their hydraulic brecciation. We did not observe any correlation between the intensity of peak metamorphism and the position of the breccias. For example, the Neocomian material exposed in the western part of the Aulus basin is nearly devoid of breccias, but it displays peak temperatures of $500-600^{\circ} \mathrm{C}$. However, other evi- dence for fluid circulations may be inconspicuous (cf. Sect. 5).

- It can result from the tectonic dismembering affecting the sedimentary pile before and/or during the peak of metamorphism. This phenomenon is observed and described further east, e.g., on the southern flank of the Mouthoumet massif and on the northern flank of the Agly massif, where sedimentary formations of various ages, from the Triassic to the Aptian, are intensely truncated and/or scalped (Durand-Delga, 1964; Bessière et al., 1989; Clerc and Lagabrielle, 2014). Tectonic contacts sealed by mid-Cenomanian deposits are commonly observed between Liassic and Paleozoic, Jurassic and Paleozoic, Middle or Upper Jurassic and Triassic, Aptian and Liassic or Triassic, and Cretaceous and Paleozoic. No stratigraphic repetition of any member of the sedimentary series has been observed, and such a disposition can be interpreted as the result of a pre-midCenomanian extensional tectonics described in the literature as the pre-Cenomanian phase (Casteras, 1933; Mattauer and Proust, 1965; Durand-Delga, 1965).

- Finally, later Alpine compressive inversion of the NPZ may also be responsible for the juxtaposition of metamorphic domains of various grades.

\subsubsection{Cenomanian and younger metasediments}

By contrast, the mid-Cenomanian, Turonian and younger metasediments always display metamorphic record of lower grade with respect to the metasediments on which they lie (up to $350^{\circ} \mathrm{C}$ in the Lherz and Vicdessos area; Fig. 8). This observation indicates that their deposition was contemporaneous to or followed the extensional tectonics described above. The Cenomanian and later flyschs probably acted as a blanket on the basins, facilitating the temperature increase.

\section{Estimating the importance of fluid circulations}

The HT-LP Cretaceous metamorphism was first considered as isochemical by Ravier (1959) and Ravier and Thiebaut (1982). Evidence of fluid circulations during the metamorphism was later presented by Bernus-Maury (1984). For Bernus-Maury (1984), Golberg (1987) and Dauteuil et al. (1987), $\mathrm{CO}_{2}$ - and $\mathrm{H}_{2} \mathrm{O}$-rich fluids released by the decarbonation reaction of siliceous dolomitic limestones are responsible for local brecciation of the Mesozoic metasediments. For Minnigh et al. (1980) massive quenching of the peridotites and decarbonation reaction would have generated most of the carbonate- and peridotite-bearing breccias. However, this hypothesis does not explain (i) the sedimentary fabrics observed in some of these breccias and (ii) the syn-metamorphic foliation observed in the Mesozoic clasts 
of these breccias (Choukroune, 1980; Lagabrielle and Bodinier, 2008; Clerc et al., 2012, 2013).

Most of the scapolite occurrences are limited to the Triassic and Liassic units, which are known to be meta-evaporitic sequences (Ravier and Thiebaut 1982). Furthermore these minerals often display a chemical zonation resulting from the interaction with Na- and Cl-rich fluids, suggesting smallscale fluid circulation within the sedimentary pile (Golberg and Leyreloup, 1990). Evidence of small-scale intra-basinal fluid circulation is proposed in Fig. 14. In the NPZ, scapolite is commonly observed in various marbles where it is localized along discontinuities such as fractures (Fig. 14) or stratigraphic joints. At the Col d'Agnes (Lherz Area), lagoonal deposits of Hettangian age (Ravier and Thiebaut 1982) show a dotty aspect with numerous millimeter-size rounded pockets filled by whitish carbonates (Fig. 14c). These pockets locally interconnect and gather into veinlets and veins (Fig. 14d). We interpret this feature as evidence of percolation, segregation and migration of fluids (Fig. 14c and d). Such fluids originating from Triassic and Liassic lagoonal deposits are good candidates to explain the allochemical scapolites observed higher up in the sedimentary pile (Fig. 14a and b). According to Dauteuil and Ricou (1989), the high thermal gradient responsible for the HT-LP metamorphism $\left(>600^{\circ} \mathrm{C}-2\right.$ to 4 kbar) cannot be reached solely by thermal conduction. Instead, enhancement of the heat propagation by the fluid circulations would be efficient enough to reach gradients of more than $100^{\circ} \mathrm{C} \mathrm{km}^{-1}$. Similar gradients are reported in the Salton Sea (Muffler and White, 1969; McDowell and Elders, 1980; McDowell and Elders, 1983), where Younker et al. (1982) identified small-scale convection cells beneath an impermeable cap rock.

\section{Evaporite-enhanced thermal conduction vs. blanketing effect}

Due to their high thermal conductivity, evaporitic rocks such as anhydrite, halite or sylvite are very efficient at transferring heat to the surrounding layers. This phenomenon, referred to as the "chimney effect" in salt diapirs (Noack et al., 2010; Kaiser et al., 2011), can be responsible for positive anomalies of a few degrees in the covering and adjacent layers, and negative anomalies of a few tens of degrees in the subjacent layers (e.g., Yu et al., 1992). The temperature effect of the salt is therefore well below the temperature differences recorded in the IMZ. In the North Pyrenean realm, the abundant Triassic evaporites may hence have played a minor role in the propagation of the Cretaceous thermal anomaly. Moreover, the contrast of conductivity between limestone and evaporitic salts strongly diminishes with increasing temperatures (Table 3); e.g., the thermal conductivities of halite and calcite become equivalent around $400^{\circ} \mathrm{C}$. The pumping-up effect of the evaporites is hence probably negligible in the hottest parts of the eastern NPZ. But in the cooler central and western do- main, it may explain the focalization of the thermal anomalies around the Saraillé and Roquiague areas that are considered as former diapiric structures (Canérot 1989; Canérot and James, 1999). Moreover, facilitation of heat transfer by the evaporitic layers may also be expressed in a horizontal direction, which could explain the strong contrast of temperature observed between the Triassic evaporites and the overlying series in the Arnave, Arignac, Bonrepaux, Betchat, Salies du Salat, Gotein and Caresse-Salies du Béarn areas (Thiébaut et al., 1988, 1992). A similar process may explain the higher temperatures obtained on the Mesozoic cover (up to $494^{\circ} \mathrm{C}$ in the Triassic of the Agly Paleozoic massif) than in the massif itself $\left(351^{\circ} \mathrm{C}\right.$ in the Silurian).

By contrast, claystone, shales and organic-matter-rich sediments are characterized by remarkably low thermal conductivities in the range of $0.2-1.0 \mathrm{~W} \mathrm{~m}^{-1} /{ }^{\circ} \mathrm{C}$, lower by a factor of 2 or more than other common sedimentary rocks (Blackwell and Steele, 1989). These rocks are known to act as a thermal blanket retaining heat within the underlying rocks (Blackwell and Steele, 1989; Pollack and Cercone, 1994; Nunn and Lin, 2002). We hence propose that the organicmatter-rich black shales of the Albian-Cenomanian black flysch (Souquet et al., 1985; Debroas, 1990) may have participated in the strong thermal anomaly registered within the Albian-Cenomanian black flysch itself and in the underlying Mesozoic series. In addition, its relative impermeability due to high clay content must have consistently limited the penetration of basinal and meteoric water in the system, hence annihilating convective cooling (Boulvais et al., 2015). In contrast with the starved paleotethys margin (Manatschal and Bernoulli, 1999; Manatschal, 2004; Masini et al., 2012) and Iberian margin (Shipboard Scientific Party, 1987; Manatschal and Bernoulli, 1999; Soares et al., 2012), the Pyrenean paleomargin seems to have developed in a sedimentrich environment, favorable to a marked blanketing effect.

\section{Timing and relationship between metamorphism and magmatism}

The ages obtained on our samples confirm the contemporaneity of the Cretaceous magmatism and metamorphism in the NPZ. This is well demonstrated in the Lherz area where, with the exception of one sample, metamorphic muscovite and amphiboles provide ages similar to the amphibole age of an undeformed gabbro at $94.7 \pm 1.3 \mathrm{Ma}$. The age of about $100 \mathrm{Ma}$ reported for a single muscovite grain from this area suggests that the thermal anomaly started to develop earlier, which is in accordance with the ${ }^{40} \mathrm{Ar}-{ }^{39} \mathrm{Ar}$ data reported further east in the Arignac and Trimouns places (100-109 Ma) as well as with the ${ }^{40} \mathrm{~K}-{ }^{40} \mathrm{Ar}$ age of Les Plagneaux gabbros (107-109 Ma; Montigny et al., 1986) and the U-Pb age of several metasomatic albitite bodies in the eastern Pyrenees (117-98 Ma; Fallourd et al., 2014, and references therein). In the Moncaup area, the magmatic, metamorphic and hy- 

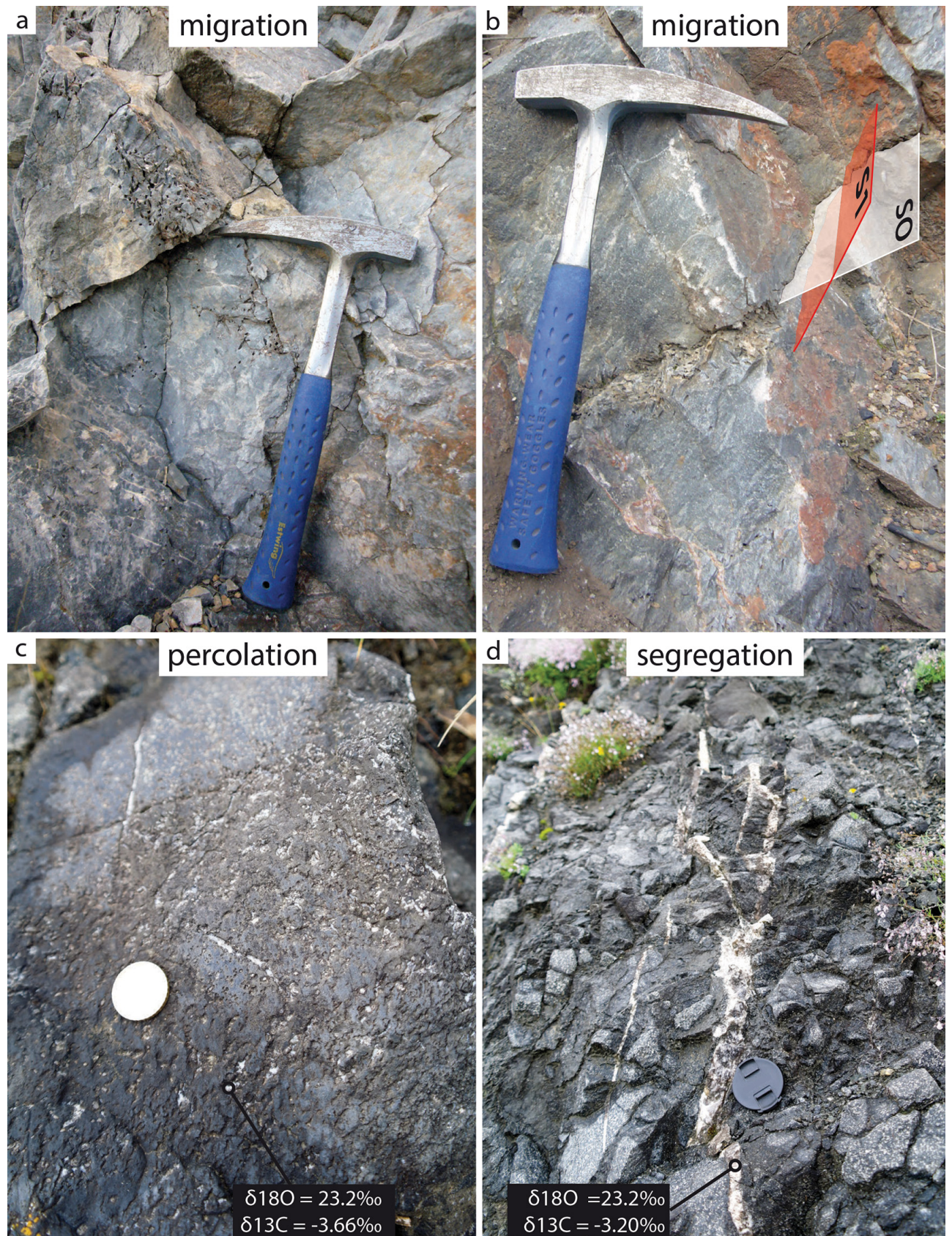

Figure 14. Top: Field indications of fluid circulations in the Urgonian marbles near Estagel (Bas-Agly syncline). Centimeter-long scapolite ghosts are found in the immediate vicinity of fractures (a) or interbeds (b). Note that the former scapolite crystals show no preferential orientation, whereas the marble presents a clear ductile foliation responsible for the stretching and flattening of fossils. In this case, the fluid circulation hence postdates the main hot deformation event. Bottom: Percolation (c) and segregation (d) of fluids in the Triassic metaevaporites of the Col d'Agnes (Aulus basin). Such meta-evaporitic material is a potential source of Na- and Cl-rich fluids responsible for the precipitation of scapolites.

drothermal activity is restricted to a shorter period as indicated by ${ }^{40} \mathrm{Ar}-{ }^{39} \mathrm{Ar}$ and $\mathrm{U}-\mathrm{Pb}$ ages close to $100 \mathrm{Ma}$.

Alteration and recrystallization of some of the NPZ Cretaceous alkaline magmatic rocks by the HT-LP metamorphism indicate that magmatism cannot account for the regional thermal anomaly (Azambre, 1967; Azambre et al., 1971, 1992;
Azambre and Rossy, 1976; Ternet et al., 1997; Azambre and Monchoux, 1998). Consistently, the present-day distribution of the Cretaceous magmatic rocks is clearly not correlated to the distribution of the isograds. For example, magmatism is abundant in the cold Mauléon basin whereas it is scarce in the Lherz or Boucheville areas. 
Table 3. Thermal conductivity of calcite, halite and pure water at $0,50,100,200,300$ and $400^{\circ} \mathrm{C}$, after Clark (1966) and Clauser and Huenges (1995).

\begin{tabular}{lrrrrrr}
\hline & \multicolumn{7}{c}{ Thermal conductivity $\left(\mathrm{W} \mathrm{m}^{-1}{ }^{\circ} \mathrm{C}^{-1}\right)$} \\
& $0{ }^{\circ} \mathrm{C}$ & $50{ }^{\circ} \mathrm{C}$ & $100{ }^{\circ} \mathrm{C}$ & $200{ }^{\circ} \mathrm{C}$ & $300{ }^{\circ} \mathrm{C}$ & $400{ }^{\circ} \mathrm{C}$ \\
\hline Calcite & 3.48 & 3.00 & 3.72 & 2.37 & 2.16 & 2.06 \\
& 4.00 & 3.4 & 2.99 & 2.55 & 2.29 & 2.13 \\
Halite (crystal) & 6.11 & 5.02 & 4.21 & 3.12 & 2.49 & 2.09 \\
Halite (Rock) & $>6.65$ & $>6.57$ & $>6.57$ & $>4.80$ & $>3.67$ & $>2.98$ \\
& $<8.25$ & $<4.80$ & $<4.80$ & $<3.67$ & $<2.98$ & $<2.47$ \\
Pure water & 0.56 & - & 0.66 & - & - & - \\
\hline
\end{tabular}
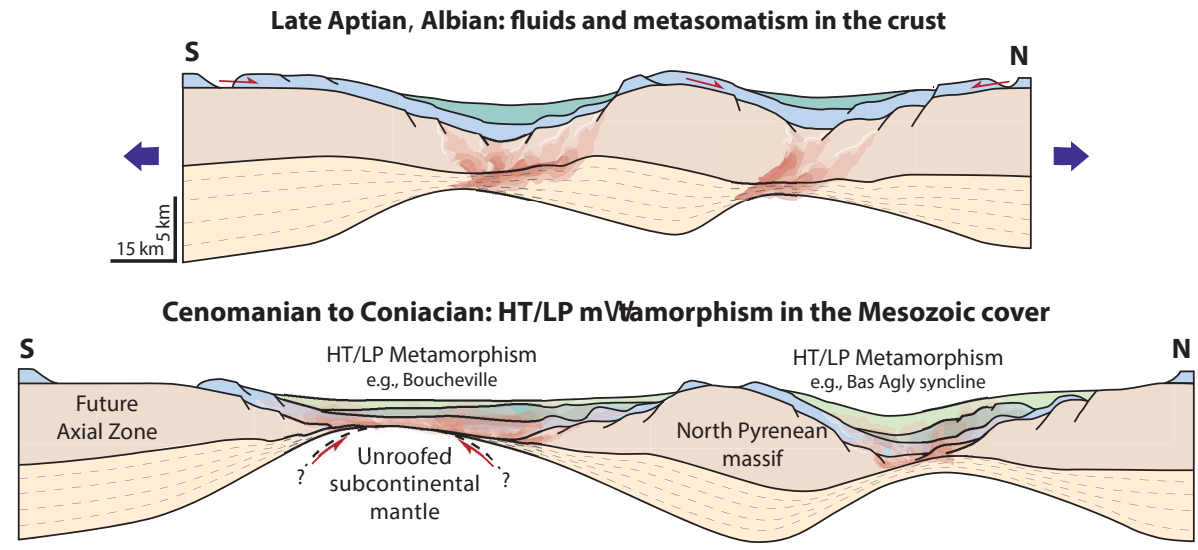

Figure 15. Schematic representation of the evolution of the Pyrenenean Cretaceous metamorphism and magmatism in response to crustal extension. Top: early hydrothermal-dominated phase affecting the crystalline basement. Down: Phase of HT-LP metamorphism, occurring mainly in the pre- and syn-rift sedimentary cover, in response to the attenuation of the continental crust.

\section{Hot versus cold margins?}

Direct access to the present-day passive margin is limited by thick sedimentary deposits, and information about the thermal history of the margin is scarcely gathered. The use of fossil margins exposed in mountain belts offers a unique opportunity to study the metamorphic imprint of the extension. However, when not overprinted by the subduction metamorphism, the Alpine analog indicates only low-grade metamorphism. At present, very few examples of hot passive margin presenting evidence of exhumed subcontinental mantle or deep crust have been reported. In the Zagros Mountains, mapping reveals that pre-rift cover and mantle were superposed early in the Kermanshah ophiolite (Wrobel-Daveau et al., 2010), where high temperatures are recorded in the Mesozoic sediments along their contact with the peridotites (Hall, 1980). In the Zagros of Iraq, Jassim et al. (1982) described a similar metamorphism affecting sediments close to exhumed ultramafic rocks with temperatures up to $750^{\circ} \mathrm{C}$ over $2.5 \mathrm{~km}$ thickness. In the light of our results, we propose a distinction between "cold" Iberian or Alpine-type passive margins and "hot" Pyrenean -type margins. The cause of this thermal variability along passive margins is still unclear. I could be explained by several factors such as the kinematic context (transtension versus extension), the mantle dynamics (hot versus cold mantle), the sedimentary input or the extension rate.

\section{Conclusions}

In this work, we measured more than 100 RSCM peak temperatures relevant to the HT-LP metamorphism in the North Pyrenean Zone, and we report 19 new ${ }^{40} \mathrm{Ar}-{ }^{39} \mathrm{Ar}$ and ages from metamorphic and magmatic samples.

Our results are in full agreement with previous data and confirm the first-order link between the metamorphism and the Cretaceous crustal thinning. The primary link between mantle exhumation and thermal anomaly is particularly well illustrated by our new data set in the Arguenos-Moncaup area.

At the scale of the whole NPZ, we observe a clear increase of the temperatures from west to east that could be explained by a combination of several scenarios: (i) the whole domain underwent the same HT-LP metamorphism, but later tectonic inversion led to underthrusting and burial of the highest-grade rocks in the western part of the NPZ. (ii) The thermal zonation may be a consequence of the lateral vari- 
ation of the kinematics along the NPZ, implying a dominant $\mathrm{N}-\mathrm{S}$ extension in the west and a NW-SE transtension in the east, leading to enhanced thermal gradients with variable hydrothermal circulation patterns. (iii) The intensity of the HT metamorphism seems correlated to the rising velocity of the exhumed peridotites. (iv) The lateral variability of the thermal anomaly along the NPZ may be related to the scissors-shape opening of the extended domain during the Cretaceous. Isotherms may have been more spaced in the wider western NPZ than in the narrower eastern NPZ.

Different parameters may have played a role in the propagation of the metamorphism. We suggest that the circulation of fluids originating from the basin rocks themselves may have relayed the thermal anomaly across the sedimentary column (Fig. 14). In addition, it is noteworthy that the pre- and syn-rift material of the NPZ is generally limited at its base by the highly conductive evaporites of the Triassic and at its top by the thick and insulating organic-rich AlbianCenomanian black flysch. This salt effect and thermal blanket effect of the organic-rich black flysch probably facilitated the elevation of the temperatures.

Fig. 15 proposes a conceptual model in which the continental crust is first weakened, then thinned and altered by hydrothermal circulations during the upper Aptian-Albian. This first phase led to the extension and thinning of the continental crust where the North Pyrenean massifs constitute crustal boudins. During the Cenomanian to Coniacian, the hyper-thinned domains opened on each side of these boudins concentrated most of the thermal anomaly in relation to the development of thick sedimentary basins.

The presence of a long-lasting HT-LP metamorphic event along the Pyrenean paleomargins has to be taken into consideration when analyzing the thermo-structural history of passive margins. Metamorphic events of this kind has never yet been described along the fossil margins exposed within the Alpine or Appalachian-Caledonian orogens. This bears important consequences regarding (i) the variability of the thermal regime that we may expect at the foot of worldwide passive margins and (ii) the variability of structural style resulting from this changing thermicity.

\section{The Supplement related to this article is available online at doi:10.5194/se-6-643-2015-supplement.}

Author contributions. C. Clerc conducted and interpreted the structural and a part of the thermometric data and wrote most of the paper; A. Lahfid brought his expertise in RSCM and conducted part of the analysis; P. Monié conducted the ${ }^{40} \mathrm{Ar}-{ }^{39} \mathrm{Ar}$ dating; Y. Lagabrielle, C. Clerc and J. C. Ringenbach directed C. Clerc's $\mathrm{PhD}$ and as such proposed the extensive use of the RSCM on the NPZ. M. Poujol conducted U-Th dating on titanite from ArguenosMoncaup albitites; P. Boulvais contributed ideas on the crustal distribution of the HT-LP metamorphism. E. Masini participated in a sampling campaign in the western NPZ. M. de St Blanquat furnished some of the ductile lineation measurements from the eastern NPZ. All authors contributed intellectually to the paper.

Acknowledgements. This work was made possible thanks to the CNRS and TOTAL S.A. through a joint PhD grant to C. Clerc. We are grateful to B. Goffé, who encouraged the application of the Raman thermometry to the NPZ.

Edited by: D. J. J. van Hinsbergen

\section{References}

Albarède, F. and Michard-Vitrac, A.: Age and significance of the North Pyrenean metamorphism, Earth Planet. Sci. Lett., 40, 327 332, doi:10.1016/0012-821X(78)90157-7, 1978a.

Albarède, F. and Michard-Vitrac, A.: Datation du métamorphisme des terrains secondaires des Pyrénées par des méthodes Ar-Ar et $\mathrm{Rb}-\mathrm{Sr}$. Ses relations avec les péridotites associées, B. Soc. Geol. Fr., XX, 681-688, doi:10.1016/ 0012-821X(78)90157-7, 1978b.

Andersen, T. B., Corfu, F., Labrousse, L., and Osmundsen, P.T.: Evidence for hyperextension along the pre-Caledonian margin of Baltica, J. Geol. Soc., 169, 601-612, doi:10.1144/001676492012-011, 2012.

Azambre, B.: Sur les roches intrusives sous-saturées du Crétacé des Pyrénées, Thèse, Université Paris 6, Paris, 1967.

Azambre, B. and Monchoux, P.: Métagabbros amphiboliques et mise en place crustale des lherzolites des Pyrénées (France), Comptes Rendus de l'Académie des Sciences, 327, 9-15, doi:10.1016/S1251-8050(98)80012-8, 1998 (in French).

Azambre, B. and Rossy, M.: Le magmatisme alcalin d'âge crétacé dans les Pyrénées occidentales; ses relations avec le métamorphisme et la tectonique, B. Soc. Geol. Fr., 7, 1725-1728, 1976.

Azambre, B., Ravier, J., and Thiebaut, J.: A propos du phénomène de dipyrisation des roches éruptives d'âge secondaire des Pyrénées, C. R. Sceances. Acad. Sci. Ser. D, 272, 2137-2139, 1971.

Azambre, B., Rossy, M., and Lago, M.: Caractéristiques pétrologiques des dolérites tholéitiques d'âge triasiques (ophites) du domaines pyrénéen, B. Mineral., 110, 379-96, 1987.

Azambre, B., Rossy, M., and Albarède, F.: Petrology of the alkaline magmatism from the Cretaceous North-Pyrenean rift zone (France and Spain), Eur J Miner., 4, 813-834, 1992.

Barrère, P., Bouquet, C., Debroas, E.-J., Pélissonier, H., Peybernès, B., Soulé, J.-C., Souquet, P., and Ternet, Y.: Carte géol. France (1/50 000), feuille Arreau (1072), 1984a.

Barrère, P., Bouquet, C., Debroas, E.-J., Pélissonier, H., Peybernès, B., Soulé, J.-C., Souquet, P., and Ternet, Y.: Notice explicative, Carte géol. France (1/50 000), feuille Arreau (1072), BRGM éditions., BRGM, Orléans, 1984b.

Bernus-Maury, C.: Etude des paragéneses caractéristiques du métamorphisme mésozoïque dans la partie orientale des Pyrénées, Thèse, Paris, 1984.

Bessière, G., Bilotte, M., Crochet, B., Peybernès, B., Tambareau, Y., and Villatte, J.: Notice explicative, Carte géol. France (1/50 000), feuille Quillan (1077), BRGM éditions, BRGM, Orléans, 1989. 
Beyssac, O., Goffé, B., Chopin, C., and Rouzaud, J. N.: Raman spectra of carbonaceous material in metasediments: a new geothermometer, J. Metamorph. Geol., 20, 859-871, doi:10.1046/j.1525-1314.2002.00408.x, 2002.

Blackwell, D. D. and Steele, J. L.: Thermal Conductivity of Sedimentary Rocks: Measurement and Significance, in: Thermal History of Sedimentary Basins, edited by: Naeser, N. D. and McCulloh, T. H., Springer, New York, available at: http://link.springer.com.biblioplanets.gate.inist.fr/ chapter/10.1007/978-1-4612-3492-0_2 13-36, 1989.

Bodinier, J.-L., Fabriès, J., Lorand, J.-P., and Dupuy, C.: Geochemistry of amphibole pyroxenite veins from the Lherz and Freychinède ultramafic bodies (Ariège, French Pyrenees), B. Mineral., 110, 345-358, 1987.

Boillot, G., Winterer, E. L., Meyer, A. W., Baltuck, M., Bergen, J. A., Comas, M. C., Davies, T. A., Dunham, K., Evans, C. A., Girardeau, J., Goldberg, D., Jansa, L. F., Johnson, J. A., Kasahara, J., Loreau, J.-P., Luna, E., Moullade, M., Ogg, J., Sarti, M., Thurow, J., and Williamson, M. A.: Proceedings of the Ocean Drilling Program, 103 Initial Reports, Vol. 103, Ocean Drilling Program, doi:10.2973/odp.proc.ir.103.1987, available at: http:// www-odp.tamu.edu/publications/103_IR/103TOC.HTM, 1987.

Boirie, J.-M.: Etude sédimentologique des Poudingues de Mendibelza (P.A.), Ph.D. thesis, Univ. de Toulouse, Toulouse, France, 1981.

Boirie, J.-M. and Souquet, P.: Les poudingues de Mendibelza: dépôTs de cônes sous-marins du rift Albien des Pyrénées, B. Cent. Rech. Expl., 6, 405-435, 1982.

Boulvais, P., de Parseval, P., D'Hulst, A., and Paris, P.: Carbonate alteration associated with talc-chlorite mineralization in the eastern Pyrenees, with emphasis on the St. Barthelemy Massif, Mineral. Petrol., 88, 499-526, doi:10.1007/s00710-006-0124-x, 2006.

Boulvais, P., Ruffet, G., Cornichet, J., and Mermet, M.: Cretaceous albitization and dequartzification of Hercynian peraluminous granite in the Salvezines Massif (French Pyrénées), Lithos, 93, 89-106, doi:10.1016/j.lithos.2006.05.001, 2007.

Boulvais, P.: O, C and Sr isotopic constraints on vein formation during the North Pyrenean Metamorphism, Compte Rendus Géosciences, accepted, 2015.

Canérot, J.: Rifting eocrétacé et halocinèse sur la marge ibérique des Pyrénées Occidentale (France), Conséquences structurales, B. Cent. Rech. Expl., 13, 87-99, 1989.

Canérot, J. and James, V.: Diapirism and post-Triassic structural development of the western Pyrenees and southern Aquitaine, Eclogae. Geol. Helv., 92, 63-72, 1999.

Carracedo, M., Larrea, F. J., and Alonso, A.: Estructura y organización de las coladas submarinas: características de las lavas almohadilladas de edad cretácica que afloran en la Cordillera Vasco- Cantábrica, Estud. Geológicos, 55(5-6), 45-53, 1999.

Castanares, L. M., Robles, S., and Vicente Bravo, J. C.: Distribution estratigrafica de los episodios volcanicos submarinos del Albiense-Santoniense en la Cuenca Vasca (sector GernikaPlentzia, Bizkaia), Geogaceta, 22, 43-46, 1997.

Castanares, L. M., Robles, S., Gimeno, D., and Vicente Bravo, J. C.: The Submarine Volcanic System of the Errigoiti Formation (Albian-Santonian of the Basque-Cantabrian Basin, Northern Spain): Stratigraphic Framework, Facies, and Sequences, J. Sediment. Res., 71, 318-333, doi:10.1306/080700710318, 2001.
Casteras, M.: Recherches sur la structure du versant nord des Pyrénées centrales et orientales, Serv Carte Géol Fr., XXXVII(189), 515p., 1933.

Casteras, M., Canérot, J., Paris, J.-P., Tisin, D., Azambre, B., and Alimen, H.: Carte géol. France (1/50 000), feuille Oloron-SainteMarie (1051), 1970.

Chevrot, S., Villaseñor, A., Sylvander, M., Benahmed, S., Beucler, E., Cougoulat, G., Delmas, P., de Saint Blanquat, M., Diaz, J., Gallart, J., Grimaud, F., Lagabrielle, Y., Manatschal, G., Mocquet, A., Pauchet, H., Paul, A., Péquegnat, C., Quillard, O., Roussel, S., Ruiz, M., and Wolyniec, D.: High-resolution imaging of the Pyrenees and Massif Central from the data of the PYROPE and IBERARRAY portable array deployments, J. Geophys. Res. Solid Earth, 119, 6399-6420, doi:10.1002/2014JB010953, 2014.

Chevrot, S., Sylvander, M., Diaz, J., Ruiz, M., Paul, A., and the Pyrope working group: The Pyrenean architecture as revealed by teleseismic P-to-S converted waves recorded along two dense transects, Geophys. J. Int., 200, 1094-1105, doi:10.1093/gji/ggu400, 2015.

Chew, M. and Van Staal, C. R.: The ocean-continent transition zones along the Appalachian-Caledonian margin of Laurentia: exemples of large-scale hyperextension during the openning of the Iapetus Ocean, Geosci. Can., 41, doi:10.12789/geocanj.2014.41.040, 2014.

Choukroune, P.: Relation entre tectonique et métamorphisme dans la zone nord-pyrénéenne centrale et orientale, B. Soc. Geol. Fr., XIV, 3-11, 1972.

Choukroune, P.: Structure et Evolution Tectonique de la Zone Nord Pyrénéenne: Analyse de la déformation dans une Portion de Chaîne à Schistosité Subverticale, Mem. Soc. Geol. Fr. Ser., 127, 176 pp., 1976.

Choukroune, P.: Comment and reply on "Quenching: An additional model for emplacement of the Iherzolite at Lers (French Pyrenees)", Geology, 8, 514-515, doi:10.1130/00917613(1980)8<514:CAROQA>2.0.CO;2, 1980.

Choukroune, P. and Mattauer, M.: Tectonique des plaques et Pyrénées: Sur le fonctionnement de la faille transformante nordPyrénéenne; comparaisons avec les modèles actuels, B. Soc. Geol. Fr., 20, 689-700, 1978.

Choukroune, P. and Séguret, M.: Carte structurale des Pyrénées, 1973.

Clark, S. P.: Thermal conductivity. Handbook of physical constants, rev. ed. Geol. Soc. Am. Mem. 97, 460-482, 1966.

Clauser, C. and Huenges, E.: Thermal Conductivity of Rocks and Minerals, in Rock Physics and Phase Relations, edited by: T. J. Ahrens, 105-126, American Geophysical Union, available at: http://onlinelibrary.wiley.com/doi/10.1029/RF003p0105/ summary, 1995.

Clerc, C. and Lagabrielle, Y.: Thermal control on the modes of crustal thinning leading to mantle exhumation, Insights from the Cretaceous Pyrenean hot paleomargins, Tectonics, 2013TC003471, doi:10.1002/2013TC003471, 2014.

Clerc, C., Lagabrielle, Y., Neumaier, M., Reynaud, J.-Y., and SaintBlanquat, M.: Exhumation of subcontinental mantle rocks: evidence from ultramafic-bearing clastic deposits nearby the Lherz peridotite body, French Pyrenees, Bull. Soc. Geol. Fr., 183, 443459, doi:10.2113/gssgfbull.183.5.443, 2012.

Clerc, C., Boulvais, P., Lagabrielle, Y., and Blanquat, M.: Ophicalcites from the northern Pyrenean belt: a field, petrographic and 
stable isotope study, Int. J. Earth Sci., 1-23, doi:10.1007/s00531013-0927-z, 2013.

Conquéré, F.: Les pyroxénolites à amphibole et les amphibololites associées aux lherzolites du gisement de Lherz (Ariège, France): un exemple du rôle de l'eau au cours de la cristallisation fractionnée des liquides issus de la fusion partielle de lherzolites, Contrib. Mineral. Petrol., 33, 32-61, doi:10.1007/BF00373793, 1971.

Costa, S. and Maluski, H.: Use of the 40Ar-39Ar stepwise heating method for dating mylonite zones: An example from the St. Barthélémy massif (Northern Pyrenees, France), Chem. Geol. Isot. Geosci. Sect., 72(2), 127-144, doi:10.1016/01689622(88)90061-9, 1988

Dauteuil, O., and Ricou, L. E.: Une circulation de fluides de haute température à l'origine du métamorphisme crétacé nordPyrénéen, Geodin. Acta, 3, 237-250, 1989.

Dauteuil, O., Raymond, D., and Ricou, L. E.: Brèches de fracturation hydraulique dans la zone métamorphique des pyrénées, exemple à l'Est du Saint-Barthélemy., C. R. Acad. Sci. Paris Ser. II, 304, 1025-1028, 1987.

Debeaux, M. and Thiébaut, J.: Les affleurements du socle paléozoique entre les massifs de la Barousse et de Milhas, Bull. Soc. Hist. Nat. Toulouse, 93, 522-528, 1958.

Debroas, E.-J.: Sédimentogenèse et position structurale des flyschs crétacés du versant nord des Pyrénées centrales, Bull. Bur. Rech. Géol. Min., I, 305-320, 1976.

Debroas, E.-J.: Evolution de la fosse du flysch ardoisier de l'albien supérieur au Sénonien inférieur (zone interne métamorphique des Pyrénées navarro-langudociennes), Bull. Soc. Géol. Fr., 20, 639-648, 1978.

Debroas, E.-J.: Le flysch à fucoïdes d'Uchentein témoin d'un escarpement turono-sénonien inférieur de la paléofaille nord pyrénéenne, Pyrénées Centrales, France, Strata, 77-93, 1987.

Debroas, E.-J.: Le flysch noir albo-cenomanien témoin de la structuration albienne à sénonienne de la Zone nord-pyrénéenne en Bigorre (Hautes-Pyrénées, France), B. Soc. Geol. Fr., 8, 273 285,1990 .

Debroas, E.-J.: Le bassin du Flysch noir albo-cénomanien dans les Pyrénées centrales?: un rift à ouverture triphasée, en transtension senestre, d'extension décroissante vers l'Est et d'amplitude pluridécakilomé-trique, 2003.

Debroas, E.-J. and Azambre, B.: Des brèches aux lherzolites. La mise en place des Lherzolites dans les fossés du flysch noir albo-cénomanien de la Ballongues et d'Aulus (Zone NordPyrénéenne, Ariège), Excursion guide book, 120 pp., AGSO, 2012.

Demange, M., Lia-Aragnouet, F., Pouliguen, M., Perrot, X., and Sauvage, H.: Les syénites du castillet (massif de l'agly, pyrénées orientales, France): une roche exceptionnelle dans les pyrénées, Comptes Rendus Académie Sci. - Ser. IIA - Earth Planet. Sci., 329, 325-330, doi:10.1016/S1251-8050(00)88582-1, 1999.

Dinarès-Turell, J., and Garcia-Senz, J.: Remagnetization of Lower Cretaceous limestones from the southern Pyrenees and relation to the Iberian plate geodynamic evolution, J. Geophys. Res. Solid Earth, 105, 19405-19418, doi:10.1029/2000JB900136, 2000.

Durand-Delga, M.: Remarques sur la stratigraphie et la structure du Mésozoïque situé entre Estagel et Perpignan (PyrénéesOrientales), Comptes Rendus Académie Sci. Paris, 259, 837840, 1964.
Durand-Delga, M.: Au sujet de la phase anté-cénomanienne à l'Est de Quillan, C. R. Somm. Soc. Geol. Fr., 2, 61-62, 1965.

Fabriès, J., Lorand, J.-P., Bodinier, J.-L., and Dupuy, C.: Evolution of the Upper Mantle beneath the Pyrenees: evidence from orogenic spinel lherzolite massifs, J. Petrol., 2, 55-76, doi:10.1093/petrology/Special_Volume.2.55, 1991.

Fabriès, J., Lorand, J.-P., and Bodinier, J.-L.: Petrogenetic evolution of orogenic lherzolite massifs in the central and western Pyrenees, Tectonophysics, 292, 145-167, doi:10.1016/S00401951(98)00055-9, 1998.

Fallourd, S., Poujol, M., Boulvais, P., Paquette, J.-L., Blanquat, M. de S., and Rémy, P.: In situ LA-ICP-MS U-Pb titanite dating of $\mathrm{Na}-\mathrm{Ca}$ metasomatism in orogenic belts: the North Pyrenean example, Int. J. Earth Sci., 103, 667-682, doi:10.1007/s00531013-0978-1, 2014.

Froitzheim, N., and Eberli, G. P.: Extensional detachment faulting in the evolution of a Tethys passive continental margin, Eastern Alps, Switzerland, Geol. Soc. Am. Bull., 102(9), 1297-1308, doi:10.1130/0016-7606(1990)102< 1297:EDFITE> 2.3.CO;2, 1990.

Garcia-Senz, J.: Cuencas extensivas del Cretacico Inferior en los Pireneos Centrales - formacion y subsecuente inversion, $\mathrm{PhD}$ Thesis, University of Barcelona, Barcelona, Spain., 2002.

Garrido-Megias, A., and Rios, L. M.: Sìntesis geològica del Secundario y Terciario entre los rìos Cinca y Segre (Pirineo Central de la vertiente surpirenaica, provincias de Huesca y Lerida), Bol Geol Min., 83, 1-47, 1972.

Golberg, J.-M.: Le métamorphisme mésozoïque dans la partie orientale des Pyrénées: relation avec l'évolution de la chaîne au Crétacé., Doc. Trav. Centre Geol. Geophys. Montpellier., Université des Sciences et Techniques du Languedoc, Montpellier., 1987.

Golberg, J.-M., and Leyreloup, A.-F.: High temperature-low pressure Cretaceous metamorphism related to crustal thinning (Eastern North Pyrenean Zone, France), Contrib. Mineral. Petrol., 104, 194-207, 1990.

Golberg, J.-M., and Maluski, H.: Données nouvelles et mise au point sur l'âge du métamorphisme pyrénéen., C. R. Acad. Sci. Paris, 306, 429-435, 1988.

Handy, M. R., M. Schmid, S., Bousquet, R., Kissling, E., and Bernoulli, D.: Reconciling plate-tectonic reconstructions of Alpine Tethys with the geological-geophysical record of spreading and subduction in the Alps, Earth-Sci. Rev., 102), 121-158, doi:10.1016/j.earscirev.2010.06.002, 2010.

Henry, P., Azambre, B., Montigny, R., Rossy, M., and Stevenson, R. K.: Late mantle evolution of the Pyrenean sub-continental lithospheric mantle in the light of new 40Ar-39Ar and Sm-Nd ages on pyroxenites and peridotites (Pyrenees, France), Tectonophysics, 296, 103-123, doi:10.1016/S0040-1951(98)00139-5, 1998.

Hervouët, Y., Torné, X., Fortané, A., Duée, G., and Delfaud, J.: Resédimentation chaotique de méta-ophites et de marbres mésozoïques de la vallée du Job (Pyrénées commingeoises): Relations détritisme/métamorphisme en zone nord-Pyrénéenne, C. R. Acad. Sci., 305, 721-726, 1987.

Jammes, S., Manatschal, G., Lavier, L. L., and Masini, E.: Tectonosedimentary evolution related to extreme crustal thinning ahead of a propagating ocean: Example of the western Pyrenees, Tectonics, 28, doi:10.1029/2008TC002406, 2009.

Jammes, S., Tiberi, C., and Manatschal, G.: 3D architecture of a complex transcurrent rift system: The example of the Bay 
of Biscay-Western Pyrenees, Tectonophysics, 489, 210-226, doi:10.1016/j.tecto.2010.04.023, 2010.

Jourdan, F. and Renne, P. R.: Age calibration of the Fish Canyon sanidine 40Ar/39Ar dating standard using primary $\mathrm{K}-\mathrm{Ar}$ standards, Geochim. Cosmochim. Acta, 71, 387-402, doi:10.1016/j.gca.2006.09.002, 2007.

Kaiser, B. O., Cacace, M., Scheck-Wenderoth, M., and Lewerenz, B.: Characterization of main heat transport processes in the Northeast German Basin: Constraints from 3-D numerical models, Geochem. Geophys. Geosystems, 12, 1-7, doi:10.1029/2011GC003535, 2011.

Lacroix, A.: Les peridotites des Pyrénées et les autres roches intrusives non feldspathiques qui les accompagnent, C. R. Acad. Sci. Paris, 165, 381-367, 1917.

Lagabrielle, Y. and Bodinier, J.-L.: Submarine reworking of exhumed subcontinental mantle rocks: field evidence from the Lherz peridotites, French Pyrenees, Terra Nova, 20, 11-21, doi:10.1111/j.1365-3121.2007.00781.x, 2008.

Lagabrielle, Y., Labaume, P., and St Blanquat, M.: Mantle exhumation, crustal denudation, and gravity tectonics during Cretaceous rifting in the Pyrenean realm (SW Europe): Insights from the geological setting of the lherzolite bodies, Tectonics, 29, 1-26, doi:10.1029/2009TC002588, 2010.

Lagabrielle Y., Clerc C., Azambre B., Vauchez A., Lahfid A., and Bousquet R.: Ductile to brittle deformation of pre-rift sediments during mantle exhumation at a distal passive margin. Insigths from the geological setting of ultramafic bodies in the Lherz area, Comptes Rendus Géosciences, accepted, 2015.

Lahfid, A., Beyssac, O., Deville, E., Negro, F., Chopin, C., and Goffé, B.: Evolution of the Raman spectrum of carbonaceous material in low-grade metasediments of the Glarus Alps (Switzerland), Terra Nova, 22, 354-360, doi:10.1111/j.13653121.2010.00956.x, 2010.

Lemoine, M., Tricart, P., and Boillot, G.: Ultramafic and gabbroic ocean floor of the Ligurian Tethys (Alps, Corsica, Apennines): In search of a genetic imodel, Geology, 15, 622-625, doi:10.1130/0091-7613(1987)15_622:UAGOFO_2.0.CO;2, 1987.

López-Horgue, M. A., Owen, H. G., Rodriguez-Lázaro, J., OrueEtxebarria, Fernández-Mendiola, P. A., and Garcia-Mondéjar, J.: Late Albian-Early Cenomanian stratigraphic succession near Estella-Lizarra (Navarra, central northern Spain) and its regional and interregional correlation, Cretaceous Res., 20, 369-402, doi:10.1006/cres.1999.0162, 1999.

López-Horgue, M. A., Owen, H. G., Aranburu, A., FernandezMendiola, P. A., and Garcia-Mondéjar, J.: Early late Albian (Cretaceous) of the central region of the BasqueCantabrian Basin, northern Spain: biostratigraphy based on ammonites and orbitolinids, Cretaceous Res., 30, 385-400, doi:10.1016/j.cretres.2008.08.001, 2009.

Manatschal, G.: New models for evolution of magma-poor rifted margins based on a review of data and concepts from West Iberia and the Alps, Int. J. Earth Sci., 93, 432-466, doi:10.1007/s00531-004-0394-7, 2004.

Manatschal, G. and Bernoulli, D.: Architecture and tectonic evolution of nonvolcanic margins: Present-day Galicia and ancient Adria, Tectonics, 18, 1099-1119, 1999.
Manatschal, G. and Nievergelt, P.: A continent-ocean transition recorded in the Err and Platta nappes (eastern Switzerland), Eclogae Geol. Helvetiae, 90, 3-27, 1997.

Masini, E.: L'évolution tectono-sédimentaire syn-rift des bassins de marge passive profonde: Exemples du bassin de Samedan (Alpes centrales, Suisse) et du bassin de Mauléon (Pyrénées basques françaises), Thèse de Doctorat, Université de Strasbourg, Strasbourg, France, 2011.

Masini, E., Manatschal, G., Mohn, G., and Unternehr, P.: Anatomy and tectono-sedimentary evolution of a rift-related detachment system: The example of the Err detachment (central Alps, SE Switzerland), Geol. Soc. Am. Bull., 124, 1535-1551, doi:10.1130/B30557.1, 2012.

Masini, E., Manatschal, G., Tugend, J., Mohn, G., and Flament, J.-M.: The tectono-sedimentary evolution of a hyper-extended rift basin: the example of the Arzacq-Mauleon rift system (Western Pyrenees, SW France), Int. J. Earth Sci., 103, 1-28, doi:10.1007/s00531-014-1023-8, 2014.

Masson, D. G. and Miles, P. R.: Mesozoic seafloor spreading between Iberia, Europe and North America, Mar. Geol., 56, 279287, doi:10.1016/0025-3227(84)90019-7, 1984.

Mattauer, M.: Sur les schistosités d'âge tertiaire de la zone axiale des Pyrénées, C. R. Acad. Sci., 259, 2891-2894, 1964.

Mattauer, M.: Les traits structuraux essentiels de la chaîne des Pyrénées, Rev. Géogr Phys Géol Dyn, 10, 3-12, 1968.

Mattauer, M. and Proust, F.: Sur la présence et la nature de deux importantes phases tectoniques dans les terrains secondaires des Pyrénées orientales, C. R. Somm. Soc. Geol. Fr., fasc. 4, 132133, 1965.

McClay, K., Muñoz, J.-A., and García-Senz, J.: Extensional salt tectonics in a contractional orogen: A newly identified tectonic event in the Spanish Pyrenees, Geology, 32, 737-740, doi:10.1130/G20565.1, 2004.

McDowell, S. D. and Elders, W. A.: Authigenic layer silicate minerals in borehole Elmore 1, Salton Sea Geothermal Field, California, USA, Contrib. Mineral. Petrol., 74, 293-310, doi:10.1007/BF00371699, 1980.

McDowell, S. D. and Elders, W. A.: Allogenic layer silicate minerals in borehole Elmore Salton Sea geothermal field, California, Am. Mineral., 68, 1146-1159, 1983.

Minnigh, L. D., van Calsteren, P. W. C., and den Tex, E.: Quenching: An additional model for emplacement of the Iherzolite at Lers (French Pyrenees), Geology, 8, 18-21, doi:10.1130/00917613(1980)8<18:QAAMFE>2.0.CO;2, 1980.

Moine, B., Fortune, J. P., Moreau, P., and Viguier, F.: Comparative mineralogy, geochemistry, and conditions of formation of two metasomatic talc and chlorite deposits; Trimouns (Pyrenees, France) and Rabenwald (Eastern Alps, Austria), Econ. Geol., 84, 1398-1416, doi:10.2113/gsecongeo.84.5.1398, 1989.

Monié, P., Soliva, J., Brunel, M., and Maluski, H.: Les cisaillements mylonitiques du granite de Millas (Pyrenees, France); age Cretace $40 \mathrm{Ar} / 39 \mathrm{Ar}$ et interpretation tectonique, B. Soc. Geol. Fr., 165, 559-571, 1994.

Montigny, R., Azambre, B., Rossy, M., and Thuizat, R.: Etude K/Ar du magmatisme basique lié au Trias supérieur des Pyrénées Conséquences méthodologiques et pétrographiques, B. Mineral., 105, 673-680, 1982.

Muffler, L. J. P. and White, D. E.: Active Metamorphism of Upper Cenozoic Sediments in the Salton Sea Geother- 
mal Field and the Salton Trough, Southeastern California, Geol. Soc. Am. Bull., 80, 157-182, doi:10.1130/00167606(1969)80[157:AMOUCS]2.0.CO;2, 1969.

Nicolas, R.: Etude géochronologique et pétrostructurale des mylonites du massif de l'Agly., D.E.A., Univerrsité Montpellier 2, Montpellier, 1998.

Noack, V., Cherubini, Y., Scheck-Wenderoth, M., Lewerenz, B., Höding, T., Simon, A., and Moeck, I.: Assessment of the presentday thermal field (NE German Basin) - Inferences from 3D modelling, Chem. Erde - Geochem., 70, Supplement 3, 47-62, doi:10.1016/j.chemer.2010.05.008, 2010.

Nunn, J. A. and Lin, G.: Insulating effect of coals and organic rich shales: implications for topography-driven fluid flow, heat transport, and genesis of ore deposits in the Arkoma Basin and Ozark Plateau, Basin Res., 14, 129-145, doi:10.1046/j.13652117.2002.00172.x, 2002.

Olivet, J. L.: La cinématique de la plaque ibérique, B. Cent. Rech. Expl., 20, 131-195, 1996.

Le Pichon, X., Bonnin, J., and Sibuet, J.-C.: La faille nordPyrénéenne: Faille transformante liée à l'ouverture du Golfe de Gascogne, Comptes Rendus Académie Sci., 271, 1941-1944, 1970.

Pollack, H. N. and Cercone, K. R.: Anomalous thermal maturities caused by carbonaceous sediments, Basin Res., 6, 47-51, 1994.

Poujol, M., Boulvais, P., and Kosler, J.: Regional-scale Cretaceous albitization in the Pyrenees: evidence from in situ U-Th-Pb dating of monazite, titanite and zircon, J. Geol. Soc., 167, 751-767, doi:10.1144/0016-76492009-144, 2010.

Puigdefàbregas, C., and Souquet, P.: Tecto-sedimentary cycles and depositional sequences of the Mesozoic and Tertiary from the Pyrenees, Tectonophysics, 129, 173-203, doi:10.1016/00401951(86)90251-9, 1986.

Ravier, J.: Le métamorphisme des terrains secondaires des Pyrénées, Mem. Soc. Geol. Fr., 86, 1-250, 1959.

Ravier, J., and Thiebaut, J.: Lagunar origin of mesozoic marbles and hornfelds at the col-dagnes (ariege), C. R. Acad. Sci. Paris, 294, 127-130, 1982.

Roest, W. R., and Srivastava, S. P.: Kinematics of the plate boundaries between Eurasia, Iberia, and Africa in the North Atlantic from the Late Cretaceous to the present, Geology, 19, 613-616, doi:10.1130/0091-7613(1991)019<0613:KOTPBB>2.3.CO;2, 1991.

Rossy, M.: Contribution à l'étude du magmatisme mésozoïque du domaine Pyrénéen, I, Le Trias dans l'ensemble du domaine; II, Le Crétacé dans les provinces Basques d'Espagne, Thèse, Université de Besançon, Besançon, 1988.

Rossy, M., Azambre, B., and Albarède, F.: REE and Sr/1bNd isotope geochemistry of the alkaline magmatism from the Cretaceous North Pyrenean Rift Zone (France-Spain), Chem. Geol., 97, 33-46, doi:10.1016/0009-2541(92)90134-Q, 1992.

San Miguel de la Camara, M.: Las eruptiones y las rocas volcanicas de las Vascongadas, Munibe, 2-3, 115-130, 1952.

Sawyer, D. S., Whitmarsh, R. B., Klaus, A., Beslier, M.-O., Collins, E. S., Carmen Comas, M., Cornen, G., de Kaenel, E., de Menezes Pinheiro, L., Gervais, E., Gibson, I. L., Harry, D. L., Hobart, M. A., Kanamatsu, T., Krawczyk, C. M., Liu, L., Lofts, J. C., Marsaglia, K. M., Meyers, P. A., Milkert, D., Milliken, K. L., Morgan, J. K., Ramirez, P., Seifert, K. E., Shaw, T., Wilson, C., Yin, C., and Zhao, X.: Proceedings of the
Ocean Drilling Program, 149 Initial Reports, Ocean Drilling Program. available at: http://www-odp.tamu.edu/publications/149_ IR/149TOC.HTM, 1994.

Schärer, U., de Parseval, P., Polvé, M., and St Blanquat, M.: Formation of the Trimouns talc-chlorite deposit (Pyrenees) from persistent hydrothermal activity between 112 and $97 \mathrm{Ma}$, Terra Nova, 11, 30-37, doi:10.1046/j.1365-3121.1999.00224.x, 1999.

Schettino, A. and Turco, E.: Tectonic history of the western Tethys since the late Triassic, Geol. Soc. Am. Bull., 123, 89-105, doi:10.1130/B30064.1, 2010.

Shipboard Scientific Party: Introduction, Objectives, and Principal Results: Ocean Drilling Program Leg 103, West Galicia Margin, edited by: Boillot, G., Winterer, E. L., Meyer, A. W., Applegate, J., Baltuck, M., Bergen, J. A., Comas, M. C., Davies, T. A., Dunham, K., Evans, C. A., Girardeau, J., Goldberg, D., Haggerty, J. A., EJansa, L., Johnson, J. A., Kasahara, J., Loreau, J.-P., Luna, E., Moullade, M., Ogg, J. G., Sarti, M., Thurow, J., and Williamson, M. A., 103, Ocean Drilling Program, available at: http://www-odp.tamu.edu/publications/103_ IR/103TOC.HTM, 1987.

Sibuet, J.-C. and Collette, B.: Triple Junctions of Bay of Biscay and North-Atlantic - New Constraints, Geology, 19, 522-525, doi:10.1130/0091-7613(1991)019_0522:TJOBOB_2.3.CO;2, 1991.

Sibuet, J.-C., Ryan, W. B. F., Arthur, M. A., Barnes, R. O., Habib, D., Iaccarino, S., Johnson, D., Lopatin, B., Maldonado, A., Moore, D. G., Morgan, G. E., Réhault, J.-P., Sigal, J., and Williams, C. A.: Initial Reports of the Deep Sea Drilling Project, 47 Pt. 2, Vol. 47 Pt. 2, US Government Printing Office, University of California, available at: http://deepseadrilling.org/47_2/dsdp_ toc.htm, 1979.

Sibuet, J.-C., Srivastava, S. P., and Spakman, W.: Pyrenean orogeny and plate kinematics, J. Geophys. Res., 109, 18 pp, doi:10.1029/2003JB002514, 2004.

Soares, D. M., Alves, T. M., and Terrinha, P.: The breakup sequence and associated lithospheric breakup surface: Their significance in the context of rifted continental margins (West Iberia and Newfoundland margins, North Atlantic), Earth Planet. Sci. Lett., 355356, 311-326, doi:10.1016/j.epsl.2012.08.036, 2012.

Souche, A., Dabrowski, M., and Andersen, T. B.: Modeling thermal convection in supradetachment basins: example from western Norway, Geofluids, 14, 58-74, doi:10.1111/gfl.12042, 2014.

Souquet, P., Peybernès, B., Billotte, M., and Debroas, E.-J.: La chaîne alpine des Pyrénées, Géologie Alp., 53, 193-216, 1977.

Souquet, P., Debroas, E.-J., Boirie, J.-M., Pons, P., Fixari, G., Dol, J., Thieuloy, J.-P., Bonnemaison, M., Manivit, H., and Peybernès, B.: Le groupe du Flysch noir (albo-cénomanien) dans les Pyrénées, Bull Cent. Rech Exlpo-Prod Elf-Aquitaine Pau, 9, 183-252, 1985.

Souriau, A., Chevrot, S., and Olivera, C.: A new tomographic image of the Pyrenean lithosphere from teleseismic data, Tectonophysics, 460, 206-214, doi:10.1016/j.tecto.2008.08.014, 2008.

Srivastava, S. P., Schouten, H., Roest, W. R., Klitgord, K. D., Kovacs, L. C., Verhoef, J., and Macnab, R.: Iberian plate kinematics: a jumping plate boundary between Eurasia and Africa, Nature, 344, 756-759, doi:10.1038/344756a0, 1990.

Stacey, J. S. and Kramers, J. D.: Approximation of terrestrial lead isotope evolution by a two-stage model, Earth Planet. Sci. Lett., 26, 207-221, doi:10.1016/0012-821X(75)90088-6, 1975. 
St Blanquat, M., Lardeaux, J. M., and Brunel, M.: Petrological arguments for high-temperature extensional deformation in the Pyrenean Variscan crust (Saint Barthélémy Massif, Ariège, France), Tectonophysics, 177, 245-262, doi:10.1016/00401951(90)90284-F, 1990.

Ternet, Y., Colchen, M., Debroas, E.-J., Azambre, B., Debon, F., Bouchez, J.-L., Gleizes, G., Leblanc, D., Bakalowicz, M., Jauzion, G., Mangin, A., and Soulé, J.-C.: Notice explicative, Carte géol. France (1/50 000), feuille Aulus les Bains (1086), BRGM éditions, BRGM, Orléans, 1997.

Thiébaut, J., Debeaux, M., Durand-Wackenheim, C., Souquet, P., Gourinard, Y., Bandet, Y., and Fondecave-Wallez, M.J.: Métamorphisme et halocinèse crétacés dans les évaporites de Betchat le long du chevauchement frontal Nord-Pyrénéen (Haute-Garonne et Ariège, France), C. R. Acad. Sci. Paris, 307, 1535-1540, 1988

Thiébaut, J., Durand-Wackenheim, C., Debeaux, M., and Souquet, P.: Métamorphisme des évaporites triasiques du versant nord des Pyrénées centrales et Occidentales, Bull. Soc. Hist. Nat. Toulouse, 128, 77-84, 1992.

Tugend, J., Manatschal, G., Kusznir, N. J., Masini, E., Mohn, G., and Thinon, I.: Formation and deformation of hyperextended rift systems: Insights from rift domain mapping in the Bay of Biscay-Pyrenees, Tectonics, 33, 1239-1276, doi:10.1002/2014TC003529, 2014.

Vauchez, A., Clerc, C., Bestani, L., Lagabrielle, Y., Chauvet, A., Lahfid, A., and Mainprice, D.: Preorogenic exhumation of the North Pyrenean Agly massif (Eastern Pyrenees-France), Tectonics, 32, 95-106, doi:10.1002/tect.20015, 2013.

Vergés, J. and Garcia-Senz, J.: Mesozoic evolution and Cainozoic inversion of the Pyrenean Rift, Mém. Muséum Natl. Hist. Nat., 186, 187-212, 2001.

Vershure, R. H., Hebeda, E. H., Boelrigh, N. A., Priem, H. N. A., and Avé Lallemant, H. G.: K/Ar age of hornblende from hornblendite vein in the alpine ultramafic mass of the Etang de Lers (Ariège), French Pyrénées, Leidse Geol Meded, 42, 59-60, 1967.

Vétil, J.-Y., Lorand, J.-P., and Fabriès, J.: Conditions de mise en place des filons des pyroxénites à amphibole du massif ultramafique de Lherz (Ar!ège, France), C. R. Acad. Sci. Paris, 307, 587-593, 1988.
Vielzeuf, D. and Kornprobst, J.: Crustal splitting and the emplacement of Pyrenean lherzolites and granulites, Earth Planet. Sci. Lett., 67, 87-96, doi:10.1016/0012-821X(84)90041-4, 1984.

Vissers, R. L. M. and Meijer, P. T.: Iberian plate kinematics and Alpine collision in the Pyrenees, Earth-Sci. Rev., 114, 61-83, doi:10.1016/j.earscirev.2012.05.001, 2012a.

Vissers, R. L. M. and Meijer, P. T.: Mesozoic rotation of Iberia: Subduction in the Pyrenees?, Earth-Sci. Rev., 110, 93-110, doi:10.1016/j.earscirev.2011.11.001, 2012b.

Whitmarsh, R. B., Sawyer, D. S., Klaus, A., and Masson, D. G.: Proceedings of the Ocean Drilling Program, 149 Scientific Results, Vol. 149, Ocean Drilling Program, available at: http://www-odp. tamu.edu/publications/149_SR/149TOC.HTM, 1996.

Whitmarsh, R. B., Beslier, M.-O., Wallace, P. J., Abe, N., Basile, C., Beard, J. S., Froitzheim, N., Gardien, V., Hébert, R., Hopkinson, L. J., Kudless, K. E., Louvel, V., Manatschal, G., Newton, A. C., Rubenach, M. J., Skelton, A. D. L., Smith, S. E., Takayama, H., Tompkins, M. J., Turrin, B. D., Urquhart, E., Wallrabe-Adams, H.-J., Wilkens, R. H., Wilson, R. C. L., Wise Jr., S. W., and Zhao, X.: Proceedings of the Ocean Drilling Program, 173 Initial Reports, Vol. 173, Ocean Drilling Program, available at: http: //www-odp.tamu.edu/publications/173_IR/INTRO.HTM, 1998.

Wopenka, B. and Pasteris, J. D.: Structural characterization of kerogens to granulite-facies graphite: Applicability of Raman microprobe spectroscopy, Am. Mineral., 78, 533-557, 1993.

Wrobel-Daveau, J.-C., Ringenbach, J.-C., Tavakoli, S., Ruiz, G., Masse, P., and Frizon de Lamotte, D.: Evidence for mantle exhumation along the Arabian margin in the Zagros (Kermanshah area, Iran), Arab. J. Geosci., 3, 499-513, doi:10.1007/s12517010-0209-z, 2010.

Wynne-Edwards, H. R.: Flow folding, Am. J. Sci., 261, 793-814, doi:10.2475/ajs.261.9.793, 1963.

Younker, L. W., Kasameyer, P. W., and Tewhey, J. D.: Geological, geophysical, and thermal characteristics of the Salton Sea Geothermal Field, California, J. Volcanol. Geotherm. Res., 12, 221-258, doi:10.1016/0377-0273(82)90028-2, 1982.

Yu, Z., Lerche, I., and Lowrie, A.: Thermal impact of salt: Simulation of thermal anomalies in the gulf of Mexico, Pure Appl. Geophys., 138, 181-192, doi:10.1007/BF00878894, 1992. 